\title{
Philosophiques
}

\section{L’a, b, c de la sémiologie}

À propos de Silence, on parle : introduction à la sémiotique, par Jurgen Pesot

\section{Guy Bouchard}

Volume 7, numéro 2, octobre 1980

URI : https://id.erudit.org/iderudit/203145ar

DOI : https://doi.org/10.7202/203145ar

Aller au sommaire du numéro

Éditeur(s)

Société de philosophie du Québec

ISSN

0316-2923 (imprimé)

1492-1391 (numérique)

Découvrir la revue

Citer cet article

Bouchard, G. (1980). L’a, b, c de la sémiologie : à propos de Silence, on parle : introduction à la sémiotique, par Jurgen Pesot. Philosophiques, 7(2), 321-375. https://doi.org/10.7202/203145ar d'utilisation que vous pouvez consulter en ligne.

https://apropos.erudit.org/fr/usagers/politique-dutilisation/ 


\title{
ÉTUDES CRITIQUES
}

\section{L'A,B,C DE LA SÉMIOLOGIE}

\author{
À propos de Silence, on parle : \\ introduction à la sémiotique
}

par Jurgen Pesot, Montréal, Guérin, 1979

\author{
par Guy Bouchard
}

À qui ne serait pas déjà quelque peu familier avec Saussure, Jakobson ou Hjelmslev, même les "Éléments de sémiologie " de Roland Barthes ${ }^{1}$ risquent de demeurer obscurs. Il en est a fortiori de même pour les textes plus ambitieux, comme celui d'Umberto $\mathrm{Eco}^{2}$, ou plus systématiquement rigoureux, tel celui de Prieto ${ }^{3}$. Dès lors, l'idée d'une sorte d'introduction aux introductions à la sémiologie, destinée à tout lecteur qui désirerait s'initier à la discipline, s'imposait d'autant plus que les ouvrages s'assignant un tel objectif sont peu nombreux et parfois peu satisfaisants ${ }^{4}$. Aussi devons-nous

1. "Éléments de sémiologie ", in Communications, 4 (1964), p. $91-135$; texte repris dans Le degré zéro de l'écriture, Paris : Gonthier, Bibl. Médiations, 1965, p. 77-181. J'utiliserai cetre seconde version.

2. La structure absente. Introduction à la recberche sémiotique, Paris : Mercure de France, 1972, $447 \mathrm{p}$.

3. Messages et signaux, Paris : P.U.F., Coll. SUP, 1966, 167 p.

4. Tel est le cas de celui de Carontini et Peraya, intitulé Le projet sémiotique (Paris: Jean-Pierre Delarge, 1975), où la paraphrase ne se reconnaît pas toujours pour telle (v.g. p. 63-64, où la description du signe linguistique tel que le définit Saussure s'inspire fortement de Barthes sans le citer) et où fourmillent des inexactitudes et des approximations injustifiables. Par exemple, à propos de Buyssens, les auteurs écrivent : "Buyssens fut sans doute l'un des premiers à jeter les bases d'une sémiologie qui serait d'abord la description de tous les systèmes de communication non linguistiques» (p. 46) ; ils citent à l'appui de cette affirmation le passage suivant : "La sémiologie peut se définir comme l'étude des moyens urilisés pour influencer autrui et reconnus comme tels par celui que l'on veut influencer " (Éric Buyssens, La communication et l'articulation linguistique, Bruxelles: Presses universitaires, 1967, p. 11), - passage qui autorise d'autant moins la restriction de la sémiologie aux systèmes non linguistiques que, dans la suite du texte de Buyssens, le langage sera considéré comme une sémie parmi d'autres (v.g. p. 22, 43-56). Parlant plus loin de l'étape physiologique de la communication linguistique telle que la décrivait Saussure, Carontini et Peraya écrivent : "l'image acoustique, c'est-à-dire l'impression produite sur l'oreille, sera la base "naturelle " de toute théorie" (p. 57); pourtant, comme on le sait fort bien, l'image acoustique ne peut produire aucune impression sur l'oreille, car elle n'est pas le son matériel, mais son empreinte psychique (Ferdinand de Saussure, Cours de l'inguistique général, Paris : Payot, 1967, p. 98). 
louer Jurgen Pesot d'avoir risqué cette entreprise et de l'avoir menée à bon terme dans un ouvrage clair, bien construit, où l'on trouvera l'essentiel de l'information de base relative au domaine sémiologique ${ }^{5}$. Dans les pages qui suivent, nous nous proposons de donner un aperçu du contenu de l'ouvrage et de l'assortir de quelques suggestions susceptibles de l'améliorer ${ }^{6}$.

\section{Le domaine de la sémiotique}

1.1. Quel est l'objet de la science des signes ? est-elle vraiment une science? C'est à ces questions que tente de répondre la première partie du volume. À la première question l'Auteur apportera, dans la première section, la réponse suivante : «le domaine embrasse tous les signes, dans quelque acception que ce soit, à condition que, dans tous les cas, il y ait quelque chose à la place de quelque chose d'autre. En somme, et en première approximation, la sémiotique tourne autour de cette très vieille définition du signe : aliquid stat pro aliquo" (p. 17). La sémiologie serait donc, comme le stipule son nom même, la science des signes? L'Auteur restreint la portée de cette définition étymologique en précisant que

pour que cette relation substitutive s'établisse, il est nécessaire que quelqu'un l'interprète ainsi. En effet, c'est une personne qui remplit d'eau une bouteille de gin après en avoit pris une gorgée ; c'est encore une personne qui prédit un orage à partir de la forme des nuages ${ }^{7}$; et ce sont des personnes qui ont

5. Cf. Jurgen Pesot, Silence, on parle : introduction à la sémiotique, Montréal, Guérin, 1979 , 156 p. Nos références à ce volume seront désormais intégrées au texte.

6. Comme l'ouvrage de Pesot, notre texte comportera trois parties et lui empruntera leurs titres. Le lecteur qui voudrait d'abord se faire une idée d'ensemble du volume n'aurait qu'à lire d'une traite la première section de chaque partie. Nous devons malheureusement signaler que l'ouvrage de Pesot aurait besoin d'un sérieux époussetage stylistique qui en supprimerait non seulement les coquilles typographiques (v.g., dans la Table des matières, on lit "La conversation du message en signaux", au lieu de "la conversion"), mais surtout des maladresses d'expression comme "culturalisation " à la place d'acculturation (p. 29), l'absence de " le " dans " comme on peut facilement [. . . deviner » (p. 45), le fair de parler de codes « qui seront traités" (p. 46) comme s'il s'agissait de fourrures, l'utilisation de « de " à la place de " pour » dans "l'impossibilité de deux êtres d'entrer en communion mutuelle» (p. 55), etc.

7. À propos de cet exemple, Pierre Guiraud fait une curieuse confusion (cf. La sémiologie, Paris : PUF, Que sais-je? n. 1421, 1971, p. 29) : décrétant que tout signe est en vue de la communication, il en infère que cette définition exclut les indices naturels tels les nuages annonçant la pluie, "car le ciel nuageux n'a pas l'intention de nous communiquer une information "; puis il ajoute que " ces indices peuvent être utilisés comme signes : ainsi les nuages des cartes météorologiques de la télévision "; or, en ce dernier cas, il ne s'agit plus de nuages réels, ni donc d'indices, mais de “ nuages " dessinés qui sont d'emblée des « signes». 
boudé tel modèle d'automobile parce que, dit-on, la grille du capot avait la forme ovale d'une vulve. J'exclus donc ici, à dessein, d'une part le domaine de la "zoo-sémiotique ", d'autre part l'ensemble des signaux qui, en tant que tels, n'ont pas besoin d'interprètes humains (p. 17).

Comment classer les signes ainsi délimités? Après avoir récusé les classifications fondées sur l'aspect matériel du signe (machines, mains, encre, tissus, sons, etc.) ainsi que sur le canal de transmission ou le type de sensation produite (audition, vision, etc.), l'Auteur se rabat sur l'énumération des dix-huit catégories de phénomènes sémiologiques proposée par Éco ${ }^{8}$. Mais tous ces phénomènes ressortissent par ailleurs à quelque autre science déjà établie, de sorte que l'objet de la sémiologie devient protéiforme et risque de se dissoudre si l'on ne se résigne à opérer un choix parmi ses objets possibles et à préciser l'angle sous lequel on les abordera : c'est pourquoi la section suivante rappelle l'opposition entre sémiologie de la communication et sémiologie de la signification, tandis que la dernière section esquisse la voie suggérée par Éco, pour qui tout phénomène culturel doit être considéré comme un phénomène de communication ; cette position, ajoute l'Auteur, suggère la possibilité d'interpréter la culture contemporaine comme un phénomène de communication de masse, mais au risque de ressusciter un pansémiologisme pour lequel, cette fois, tout serait communication. À cet impérialisme des mass media s'oppose celui de Barthes, pour qui toute pratique signifiante s'imprègne de linguistique, tandis qu'entre ces deux extrêmes se faufile la voie du symbolisme empruntée par Todorov et par Sperber.

Il importe à présent de poser de nouveau cette question cruciale : la sémiotique (sémiologie) est-elle une science, une discipline? Une chose est certaine: elle n'est pas une discipline unitaire comme en sont nos disciplines traditionnelles, la physique, la statistique, la théologie. Tout se passe comme si diverses disciplines s'étaient trouvées des intérêts communs, préparant ainsi la voie à des études interdisciplinaires. À l'instar de toute approche interdisciplinaire, la sémiotique serait alors caractérisée plus par son point de vue que par son objet et sa méthode (p. 32).

8. Cf. La structure absente, p. 14-19. Nous reviendrons sur cette liste dans nos commentaires. 
Mais, contrairement à l'écologie, la sémiotique ne s'est pas encore imposée comme une nouvelle discipline, et l'Auteur reprend à son compte l'affirmation de Todorov : "On voit que la sémiotique [...] reste pour l'instant un ensemble de propositions, plus qu'un corps de doctrine constitué?."

1.2. De cette première partie, trois thèmes principaux susciteront nos commentaires : celui de la définition du signe, celui du découpage du champ de la sémiologie et celui des principales tendances de cette discipline.

1.2.1. Nous avons vu que, selon l'Auteur, la sémiologie tournait, "en première approximation ", autour de la définition du signe : aliquid stat pro aliquo. Notons tout d'abord que cette première approximation est en fait définitive et que l'Auteur ne la révisera plus, au contraire : "Aliquid stat pro aliquo, disaient les anciens philosophes; Saussure, Bloomfield, Büchler, Hjelmslev, Peirce et tous les autres ne disent foncièrement rien de très différent $»(\mathrm{p} .70)$. Tel est également l'avis de Jakobson :

La définition médiévale du signe - aliquid stat pro aliquo que notre époque a ressuscitée, s'est montrée toujours valable et féconde. C'est ainsi que la marque constitutive de tout signe en général, du signe linguistique en particulier, réside dans son caractère double : chaque unité linguistique est bipartite et comporte deux aspects, l'un sensible et l'autre intelligible d'une part le signans (le signifiant de Saussure), d'autre part le signatum (le signifiè). Ces deux éléments constitutifs du signe linguistique (et du signe en général) se supposent et s'appellent nécessairement l'un l'autre ${ }^{10}$.

Suffit-il cependant qu'une chose tienne lieu d'une autre chose pour que celle-là soit le signe de celle-ci ? Une jambe artificielle est-elle le signe de la jambe naturelle qu'elle remplace? Le texte de Jakobson est, à cet égard, plus précis que celui de l'Auteur: le signe n'est pas une relation de substitution quelconque ${ }^{11}$, mais une relation telle que l'un des

9. Oswald Ducrot, Tzvetan Todorov, Dictionnaire encyclopédique des sciences du langage, Paris : Seuil, 1972, p. 122.

10. Roman Jakobson, Essais de linguistique générale I, Paris : Éd. de Minuit, 1968, p. 162.

11. Dans ses "Éléments de sémiologie ", Barthes est encore plus vague, puisqu'il s'en tient à une relation quelconque : "Signe s'insère en effet, au gré des auteurs, dans une série de termes affinitaires et dissemblables: signal, indice, icône, symbole, allégorie sont les principaux rivaux du signe. Posons d'abord l'élément commun à tous ces termes : ils renvoient tous à une relation entre deux relata » (p. 107). 
relata (le signans) soit sensible, et l'autre (le signatum), intelligible, les deux constituant l'unité qu'est le signe. Cette précision, cependant, suscite elle aussi certains problèmes. Par exemple, assimiler le signans au signifiant saussurien, et le signatum au signifié, c'est occulter la différence entre le son et le signifiant et transférer au second la matérialité du premier $^{12}$.

Lorsque l'on décrit la sémiologie comme étant la science des signes, il nous semble que le concept de signe devrait, même dans une introduction à la discipline, être quelque peu élaboré. Il importe, entre autres, de distinguer soigneusement, d'une part, l'ensemble de termes par rapport auxquels se définit le signe, d'autre part les éléments constitutifs du signe lui-même. Dans le premier cas, on aura des conceptions dyadiques, triadiques, tétradiques ou pentadiques du signe ${ }^{13}$. Illustrons chacune d'entre elles.

Dans son Cours de linguistique générale ${ }^{14}$, Saussure s'oppose à la conception de la langue comme nomenclature, c'est-à-dire comme liste de mots correspondant à autant de choses ; pour cette conception " naïve ", il n'y aurait donc que deux termes en présence : le signe et la chose. Saussure ajoute cependant que cette conception présuppose des idées toutes faites préexistant aux mots : il introduit ainsi un troisième terme, l'idée, par lequel cette conception n'est plus dyadique que par malformulation. Il en va de même dans le cas de la Grammaire de Port-Royal ${ }^{15}$, où l'élision apparente porte sur les choses : "Ainsi l'on peut considérer deux choses dans ces signes. La première; ce qu'ils sont par leur nature, c'est-à-dire en tant que sons et caractères. La seconde ; leur signification, c'est-àdire la manière dont les hommes s'en servent pour signifier leurs pensées. " Mais les pensées, comme l'explique expressément la suite du texte, ont trait aux choses ( Concevoir, n'est autre chose qu'un simple regard de notre esprit sur les

12. Confusion que l'on retrouve chez Barthes lorsqu'il parle de la "matérialité du signifiant " ("Éléments . . ., p. 119).

13. Nous nous en tenons aux plus importantes. En chacune de ces conceptions nous sous-entendons le fait que le signe est tel par rapport à un interprète.

14. P. 97-98.

15. Annauld et Lancelot, Grammaire générale et raisonnée de Port-Royal, Paris : Bossange et Masson, $1810,2^{e}$ éd., p. 245-246 et 268. 
choses »....), de sorte que cette conception articule en réalité trois termes.

Beaucoup plus fréquentes sont les conceptions triadiques, dont voici un échantillon :

\begin{tabular}{|c|c|c|}
\hline Augustin ${ }^{16}$ & $\begin{array}{l}\text { Ogden et } \\
\text { Richards }{ }^{17}\end{array}$ & Schaff $^{18}$ \\
\hline Signe & Symbole & Signe \\
\hline Pensée & $\begin{array}{l}\text { Pensée } \\
\text { (référence) }\end{array}$ & Pensée \\
\hline Chose & Référent & Réalité \\
\hline
\end{tabular}

Les différences entre ces conceptions sont principalement d'ordre terminologique. Nous proposons de les considérer comme des variantes de la conception "classique 》 du signe, de décrire cette conception à l'aide des termes "signe", "pensée » et « désigné », et de la représenter, suivant Ogden et Richards, par un triangle :

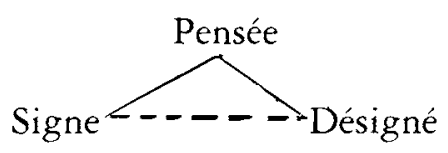

Comme exemple de la conception tétradique, prenons la Logique de Port Royal ainsi que la linguistique saussurienne. Selon les auteurs de la première,

16. "Par signe on entend une chose qui frappe extérieuremenr nos sens, mais qui fait naître en nous la pensée d'une autre chose " (Augustin, Sur la doctrine chrétienne, in Oemres complèter t. VI, Paris : Louis Vivès, 1873 , p. 470).

17. Symbols direct and organize, record and communicate. In stating what they direct and organize, record and communicate we have to distinguish as always between thoughts and things. It is thought (or, as we shall usually say, reference) which is directed and organized, and it is also thought which is recorded and communicated " (O.K. Ogden, I.A. Richards, The Meaning of Meaning, New York : Harcourt, Brace and World, s.d., p. 9).

18. "Tout objet matériel, toute propriété de cet objet ou tout événement matériel devient un signe, quand dans le processus de la communication il sert, dans le cadre du langage adopté par les personnes qui communiquent, à transmettre certaines pensées concernant la réalité, autrement dit le monde extérieur ou des expériences intérieures (affectives, esthétiques, volontaires, etc...) de l'une des personnes qui communiquent "( $A$. Schaff, Introduction à la sémantique, Paris: Anthropos, 1969, p. 161). Pour une discussion de cette définition et de la typologie des signes qui l'accompagne, of. $G$. Bouchard, "La typologie des signes selon Adam Schaff", in Laval théologique et pbilosophique, XXXIV (1978), p. 57-97. 
quand on ne regarde un certain objet que comme en représentant un autre, l'idée qu'on en a est une idée de signe, et ce premier objet s'appelle signe (. . .) Ainsi le signe enferme deux idées, l'une de la chose qui représente, l'autre de la chose représentée; et sa nature consiste à exciter la seconde par la première $^{19}$.

Nous aurions pu présenter cette théorie dans le cadre de la conception classique : dire qu'on appelle signe un objet qui en représente un autre, cela ressemble fort, en effet, à l'affirmation que le signe est une chose sensible qui fait naître la pensée d'une autre chose. Mais si l'on tient compte de la seconde partie de la définition, d'après laquelle le signe renferme deux idées : l'une de la chose qui représente, l'autre de la chose représentée, l'on peut considérer cette théorie comme mettant en relation quatre termes : la chose qui représente, l'idée de la chose qui représente, l'idée de la chose représentée et la chose représentée. Quant au signe lui-même, ici il se compose de deux éléments : l'idée de la chose qui représente et celle de la chose représentée : en termes saussuriens, le signifiant et le signifié. Ceux-ci se distinguent, chez Saussure ${ }^{20}$, d'une part, de la réalisation matérielle du signifiant ${ }^{21}$, d'autre part, de la chose, c'est-à-dire du désigné. Nous appellerons conception saussurienne cette double explicitation tétradique du signe et nous la représenterons comme suit :

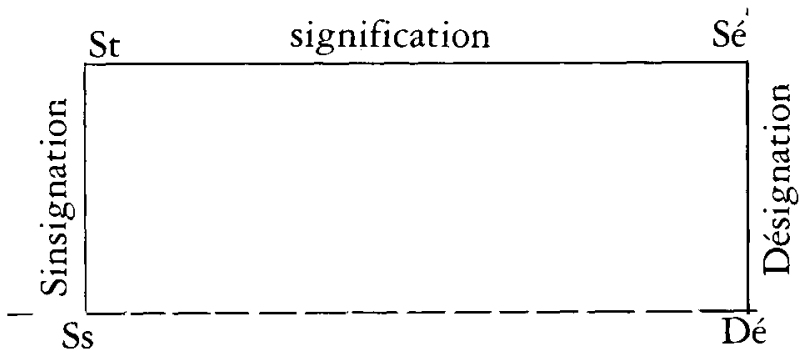

19. Arnauld et Nicole, La logique ou l'art de penser, Paris: P.U.F., 1965, p. 53.

20. Cours de linguistique générale, p. 97-100.

21. Saussure oppose expressément, dans le cas du signe linguistique, le son à l'image acoustique (signifiant). Au niveau du signe en général l'équivalent du son serait ce que Charles Morris appelait le véhicule de signe; mais comme il est difficile de construire des dérivés de cette expression, nous lui substituerons le terme sinsigne, qu'utilisait Peirce pour désigner le signe qui est un objet ou un événement individuel (Collected Papers, 8.334), et que Morris identifie ou véhicule de signe (cf. Foundations of the Theory of Signs, Chicago: The University of Chicago Press, 1938, p. 3 et 50). 
On peut considérer comme conceptions pentadiques du signe celles de Buyssens ${ }^{22}$ et de Prieto ${ }^{23}$. Schématiquement, et en suivant la terminologie de Buyssens, cette conception articule le fait perceptible ( $\mathrm{Fp}$ ), le signifiant ( $\mathrm{St})$, le signifié (Sé), l'état de conscience $(\mathrm{Ec})$ et le désigné (Dé) :

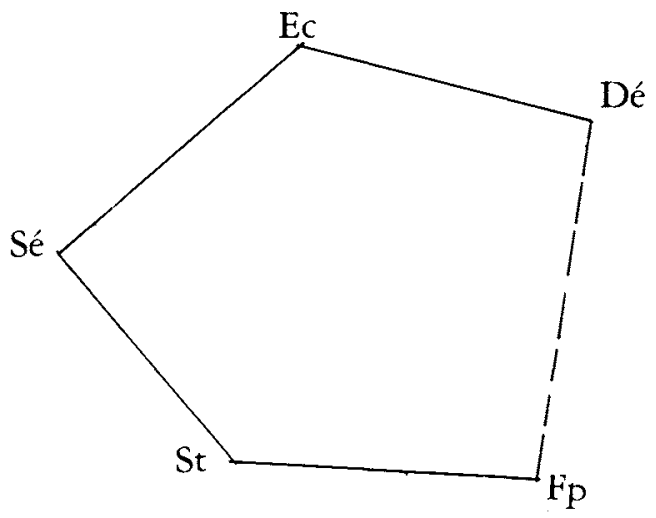

Le fait perceptible et l'état de conscience constituent ensemble l'acte sémique, tandis que le signifiant et le signifié composent le sème. Le sème étant, d'après Buyssens, une abstraction qui ne retient que les éléments fonctionnels de l'acte sémique, nous désignerons désormais la théorie de Buyssens - Prieto par l'expression " conception fonctionnelle ".

Qu'en est-il, par rapport à toutes ces conceptions du signe, de la formule aliquid stat pro aliquo? L'Auteur devrait, sinon trancher la question, du moins donner quelque idée de la complexité du problème. Il est d'ailleurs intéressant de noter que toutes ces conceptions sont fort voisines les unes des autres. Dans la mesure où le « fait perceptible » (le «signal» de Prieto) équivaut au sinsigne, la seule différence entre la conception saussurienne et la conception fonctionnelle tient à ce que celle-ci se préoccupe de l'état de conscience en tant que particularisation par le sujet du signifié. De même, le rapport entre la conception classique et la conception saussurienne deviendrait plus étroit si l'on admettait que la distinction entre

22. Les langages et le discours, Bruxelles : Office de Publicité, 1943, 96 p.; La communication et l'articulation linguistique, Bruxelles : Presses universitaires, 1967, $175 \mathrm{p}$.

23. Messages et signaux, Paris : P.U.F., 1966, 167 p. 
sinsigne et signifiant, rarement thématisée comme telle, est pourtant implicite dans la première ${ }^{24}$ : les deux conceptions articuleraient alors quatre termes et différeraient par le nombre d'éléments constitutifs du signe : dans la conception classique, le signe est une entité simple extérieure à la pensée, tandis que dans la conception saussurienne (et dans la conception fonctionnelle) le signe est une entité psychique bifaciale intégrant (l'aspect social de) la pensée.

1.2.2. À propos du découpage du champ sémiologique, nous avons vu que l'Auteur récuse les classifications fondées sur le canal de transmission ou le type de sensation, pour se rabattre sur l'énumération proposée par Umberto Eco et que l'on peut représenter par le tableau suivant ${ }^{25}$ :

Zoosémiotique (1)— sémiologie médicale (6) Anthroposémiologie

-
-
-
-
-

communication olfactive (2)

communication tactile (3)

communication gustative (4)

communication auditive :

- langues naturelles

- para-linguistique (5)

- codes musicaux (8)

communication visuelle (12)

- langues écrites, alphabets inconnus, codes secrets (10)

- kinésique et proxémique (7)

- langages formalisés (9)

- système des objets (13)

- codes culturels (15

Communication des Machines (re : langages formalisés : 9)

24. Chez Augustin, mais en des textes généralement peu connus des sémiologues, elle apparaît même explicitement : cf. G. Bouchard, "La conception augustinienne du signe selon Tzvetan Todorov", in Recherches augustiniennes XV (1980), p. 305-346. Quant à Schaff, il semble lui aussi reconnaître une différence entre le son et l'image sonore (Opus cit., p. 182).

25. Tableau que j'ai déjà utilisé dans "Les principales tendances de la sémiologie ", in Dialogue, XIV (1975) 4, p. 653. La classe « codes culturels * recouvre des phénomènes comme l'étiquette, les hiérarchies, les systèmes de parenté, etc. ; son rapport aux autres catégories du secteur anthroposémiologie ressortirait davantage si l'on regroupait 
Les chiffres correspondent aux facteurs mentionnés par Éco et au désordre de leur présentation; les facteurs non numérotés sont ceux que nous avons ajoutés pour transformer cette énumération en classification. Celle-ci, comme on peut le constater, fait une large place aux catégories sensorielles et s'expose donc aux reproches que l'Auteur adresse à ce type de regroupement : " on est obligé de créer de grandes catégories mixtes qui se révéleront peut-être plus importantes que les catégories "pures", en invalidant eo ipso le critère retenu» (p. 19) ; de plus, "nombre de phénomènes restent inclassables en termes de sensations, tout en participant à une sémiose importante : les figures du langage et le récit, les mythes et les légendes, les actes dits symboliques, les mass-média, etc. » (p. 20).

Mais ces critiques sont-elles fondées? Rien n'oblige, en effet, à limiter l'application du critère sensoriel à des formes « pures", et on voit difficilement comment la catégorie mixte « communication audio-visuelle » invaliderait le critère retenu, dont elle exprime au contraire l'une des possibilités combinatorielles ; en effet, à partir de cinq canaux sensoriels, l'on obtient $2^{5}-1$, c'est-à-dire 31 combinaisons possibles, dont cinq seulement sont «pures"; mais si l'on pose que le seul syncrétisme effectif est celui de la vue et de l'ouie, l'on retiendra une seule des combinaisons mixtes. Quant aux phénomènes "inclassables en termes de sensations", ils ne posent pas vraiment de problème: les figures du langage relèvent de la même catégorie que le langage lui-même, et des ensembles comme les récits, les mythes et les légendes peuvent être découpés en sous-ensembles correspondant à leurs modes d'expression (v.g. récit oral, récit écrit, récit audio-visuel), tout comme les mass média ${ }^{26}$.

Un dernier problème est celui de la zoosémiotique. L'Auteur exclut celle-ci de sa conception de la sémiologie,

celles-ci sous le titre "point de vue du signifiant", les "codes culturels" correspondant alors au "point de vue du signifié ", comme le laisse entendre Éco lui-même (p. 18) : "Tous ces systèmes, disparates à première vue, intéressent pourtant la communication dans la mesure où les éléments qui les composent deviennent les contenus possibles de communications verbales, visuelles, gestuelles, etc. ".

26. Ces "phénomènes » sont en fait engendrés par des classificarions reposant sur d'autres critères. 
mais il note que "pour un des sémioticiens aujourd'hui les plus en vue, Umberto Eco, les signes animaux appartiennent au domaine sémiotique » (p. 19). Effectivement, la zoosémiotique occupe la première position dans l'énumération d'Eco. Mais, après avoir proposé cette énumération, Eco entreprend de déterminer le « seuil " sémiotique, ce qui l'incite à décréter que la sémiologie "étudie les processus culturels (c'est-à-dire ceux où interviennent des agents humains qui entrent en contact sur la base de conventions sociales), comme processus de communication ${ }^{27}$ : la sémiologie préconisée par Eco exclut donc la zoosémiologie et la communication des machines, c'est une anthroposémiologie, tout comme celle de l'Auteur.

1.2.3. À propos des tendances de la sémiologie, l'Auteur rappelle d'abord l'opposition entre sémiologie de la communication et sémiologie de la signification. Appartiennent à la première tendance des auteurs comme Buyssens et Prieto. L'Auteur semble s'étonner que, dans les quelques lignes qu'il consacre à l'histoire de la sémiologie, Buyssens ne mentionne qu'un nom, celui de Rudolf Kleinpaul ${ }^{28}$; il ajoute ensuite que Buyssens précise ce qu'il faut entendre par interprétation, par signification des comportements humains et animaux, des objets et phénomènes interprétables,

dans le but de mieux les bannir et de mieux dégager le " fair sémiologique ». Selon lui, le fait interprétable ne constitue qu'un indice - que Prieto définira comme " un fait immédiatement perceptible qui nous fait connaître quelque chose à propos d'un autre qui ne l'est pas » [ . . ] L'indice n'est un fait

27. La structure absente, p. 24.

28. Voici le texte de Buyssens : "L'histoire de la sémiologie n’est pas longue. Avant Saussure, on trouve, surtout chez les logiciens, des remarques générales concernant les signes ou les symboles. Mais il faut citer en particulier "Sprache ohne Worte " de Rudolf Kleinpaul; l'auteur distingue nettement les actes dus à une authentique volonté de communiquer et les faits susceptibles d'interprécation; en outre, il apporte une collection importante de faits intéressants la sémiologie" (La communication at l'articulation linguistique, éd. de 1970 , p. 12). L'expression " avant Saussure ", en plus de préciser la portée historique du paragraphe, ajoute au moins un nom à celui de Kleinpaul. Elle est suivie d'un paragraphe mettant l'accent sur la contribution de Charles Serrus, puis d'un autre énumérant les noms de Vendryes, de Buyssens, de Barthes, de Lévi-Strauss et de Mounin. On notera par atlleurs que cet ouvrage, publié pour la première fois en 1967, reprend pour l'essentiel celui de 1943 : Les Langages $t$ t te discours. 
sémiologique que s'il est artificiel, conventionnel, produit expressément comme un moyen et perçu comme tel (p. 23).

Cette lecture de Buyssens est malheureusement trop hâtive, comme pourra nous en convaincre le passage suivant de La communication et l'articulation linguistique :

Dire que nous comprenons la signification du poing serré ou de la pâleur, c'est dire que nous devinons la cause du comportement considéré; mais dire que nous comprenons la phrase «Viens ici!" ou d'un poteau indicateur, c'est dire que nous devinons l'intention de celui qui a recouru au fait considéré. Dans le premier cas, ce que nous interprétons est un effet, dans le second un moyen ; c'est là que gît le critère qui permet de distinguer le fait sémiologique des indices (p. 17).

Comme on peut le constater, il y a signification et interprétation autant dans le cas du fait sémiologique que dans celui de l'indice, et l'indice n'est pas un fait sémiologique. La confusion entre indice et fait sémiologique est d'ailleurs imputable moins à l'Auteur qu'à Prieto, qui écrit :

Aussi le signal peut-il être défini comme un indice artificiel, c'est-à-dire comme un fait qui fournit une indication et a été produit expressément pour cela. Cf. Buyssens, Les langages et le discours, \#14, et Prieto, La sémiologie ${ }^{29}$.

Or au paragraphe 14 de Les langages et le discours, Buyssens affirme qu'il y a une caractéristique commune au "langage des faits " et à l'acte de communication : dans les deux cas le témoin d'un fait interprète celui-ci comme révélateur d'un autre fait, à savoir un état de conscience; mais ce qui caractérise l'acte de communication, c'est que le fait perceptible est réalisé volontairement pour que le témoin en reconnaisse la destination :

fait perceptible révélant

un état de conscience

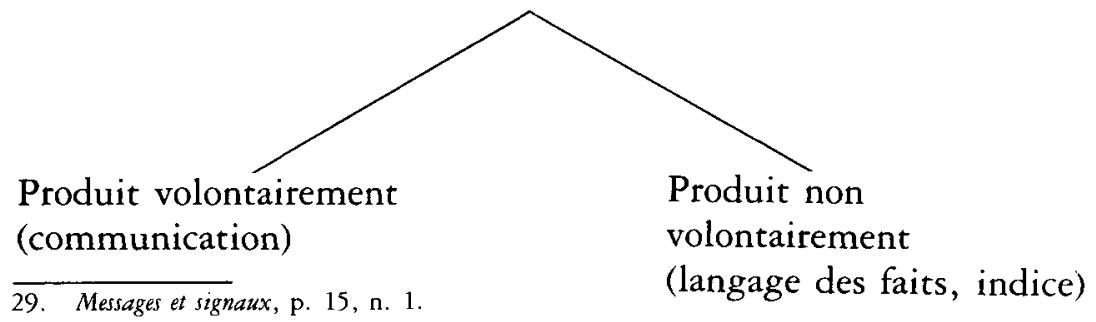


Il n'y a pas, chez Buyssens, de terme désignant ce qu'il y a de commun aux procédés de communication et aux indices. Prieto, pour combler cette lacune, emprunte le mot indice, ce qui l'oblige à remplacer l'indice de Buyssens par un indice artificiel. Pour éviter cet imbroglio, il suffirait de redonner au mot signe son acception générique et de reconnaître qu'il y a des signes qui servent à communiquer (les signaux ${ }^{30}$ ) et d'autres qui ne servent pas à communiquer (les indices).

Cette rectification terminologique offrirait au moins l'avantage de préciser l'opposition entre sémiologie de la communication et sémiologie de la signification. Nous avons vu en effet que Buyssens oppose signaux et indices; mais si l'on reformule l'opposition en termes de "signaux communicatifs » et d'indices significatifs », on risque de laisser entendre que la sémiologie de la signification aurait pour objet les indices, alors qu'il s'agit en fait d'une sémiologie des signes en général.

La triade signe, signal et indice permet de préciser plusieurs autres points.

L'Auteur parle des manuels ${ }^{31}$ qui stipulent que la sémiologie s'occupe des signes, lesquels composent les messages nécessaires à la communication humaine, et qui, par exemple dans le cas de la «communication olfactive", prennent pour exemple des phénomènes qui sont de toute évidence des indices, ce qui dilue la notion de communication (p. 23-24) : ces remarques sont tout à fait justes, sauf qu'on ne devrait pas s'en autoriser pour traiter sur le même pied ces manuels d'une part, d'autre part la tendance illustrée par Buyssens, Prieto et Mounin ; celle-ci, en effet, constitue une véritable tendance, où le mot communication reçoit une définition précise, alors que ceux-là relèvent simplement de la

30. Le terme signal est habituellement utilisé par l'Auteur pour désigner un élément physique transmis d'une entité à une autre (p. 17-28-29-40-41-43) et qui, en tant que tel, "relève de la physique et de la technologie, non de l'analyse sémiologique " (p. 40); mais, lorsqu'il parle du schisme que verrait Buyssens " entre indices significatifs et signes ( signaux") communicatifs" (p. 24), il utilise le terme signal au sens où nous le prenons et qui est celui de Prieto et de Mounin (Introduction à la sémiologie, Paris : Éd. de Minuit, 1970, p. 13-15).

31. Un seul est mentionné, celui de Toussaint (Qu'est-ce que la séminlngie?, Toulouse: Privat, 1978). 
foule d'ouvrages qui emploient les termes de base d'une discipline sans trop se soucier de rigueur (en règle générale, la co-occurrence des termes «signe " et «communication", lorsqu'elle ne s'accompagne pas de clauses restreignant la portée du premier terme, est l'indice d'un usage élargi du second).
À propos de la sémiologie de la signification, l'Auteur écrit :
il arrive qu'on restreigne le concept de la signification pour en faire le seul objet de la sémiotique. Témoin John Lyons (op. cit. ${ }^{32}$ ) qui, dans un chapitre intitulé "Semiotics", ne traite, en gros, que de la typologie des signes (et des trois parties constitutives de la sémiotique : syntaxe, sémantique, pragma- tique), alors que des notions comme communication et information, référence et dénotation, etc., sont exposées à part (p. 28).

Mais qu'elles soient exposées à part, cela signifie-t-il qu'elles soient sans rapport avec la sémiologie? Dans la préface à l'édition française de son ouvrage, Lyons écrit :

Dans les sept premiers chapitres je me suis efforcé, dans les limites qui m'étaient imparties, de situer la sémantique dans le cadre plus général de la sémiotique (définie ici comme l'étude des systèmes de signaux humains et non humains) ${ }^{33}$.

Mais comme le terme signal désigne " tout ce qui est transmis le long d'un canal de communication quelconque et peut être interprété par le récepteur comme la mise en code d'un message ${ }^{34}$, il serait assez surprenant que la notion de communication, bien que "traitée à part ", n'ait rien à voir avec la sémiologie; on en dirait tout autant de celles de référence et de dénotation ${ }^{35}$. Le point le plus important, cependant, c'est moins de savoir comment interpréter Lyons que de se demander pourquoi on parle de lui. Les principaux représentants de la sémiologie de la signification étant Lévi-

32. En fait, cet ouvrage n'a pas été mentionné dans les pages précédentes. Comme il n'apparaît pas non plus dans la bibliographie de l'Auteur, il faut patienter jusquà la page 65 pour découvrir qu'il s'agit de "Senantics, Cambr: C.U.P., 1977, p. 42-3".

33. J. Lyons, Éléments de sémantique, Paris : Larousse, 1978, p. 7

34. Ibid., p. 83.

35. Cf. p. 143, où Lyons montre le lien entre ces notions et celles de langue et de systemes sémiotiques. 
Strauss et Barthes (p. 22), on peut en effet s'étonner que ceux-ci ne soient même pas mentionnés dans la maigre page (p. 27-28) consacrée à cette tendance.

Aux deux voies que nous venons de parcourir l'Auteur en ajoute une troisième : "il me semble opportun d'exposer ce qui semble bien être une sorte de troisième voie, qui neutralise l'opposition entre communication et signification en faisant de la sémiotique une science de la culture. C'est la voie indiquée par Umberto Eco pour qui tout phénomène culturel doit être envisagé comme phénomène de communication» (p. 28). Dire que la sémiologie est la science de la culture, ce n'est pas, en effet, la même chose que de l'assimiler à une science de la communication ou à une science de la signification. Mais si l'on ajoute que «la sémiotique n'est pas seulement la science des systèmes de signes reconnus comme tels, mais la science qui étudie tous les phénomènes de culture comme s'ils étaient des systèmes de signes en se basant sur l'hypothèse qu'en réalité tous les phénomènes de culture sont des systèmes de signes, c'est-à-dire que la culture est essentiellement communication ${ }^{36}$, - où est la différence, sinon dans une confusion analogue ̀̀ celle de Toussaint entre communication et signification? Pour que la suggestion d'Eco neutralisât l'opposition entre les deux autres tendances, il faudrait que la sémiologie de la signification se situe au niveau des indices, ce qui, comme nous l'avons souligné, n'est pas le cas.

En conclusion, il nous semble que la section consacrée aux tendances de la sémiologie serait beaucoup plus éclairante si elle articulait ces tendances par rapport à une typologie embryonnaire des signes où le signe et la signification occuperaient la position générique par rapport au signal et à la communication d'une part, à l'indice et à la noncommunication d'autre part; bien entendu, les tendances devraient être identifiées moins par le fait qu'elles utilisent tel ou tel mot clef (signification, communication), que par la façon dont elles définissent ces termes en les opposant ou en ne les opposant pas.

36. La structure absente, p. 261. 


\section{Autour de la communication}

2.1. Comme l'indique son titre, la seconde partie du volume porte sur la communication.

Une première section rappelle que, étymologiquement, le terme communication appartient à une famille de mots caractérisée par les notions de changement et d'échange.

La seconde section met au point un modèle de la communication. Elle commence par la formalisation du sens étymologique de communiquer : "Échange : d'une entité $A$ passe d'abord quelque chose $(\mathrm{X})$ à une entité $\mathrm{B}$ » (p. 36). Mais ce schéma est trop général : " Il intéresse le processus de la communication dans la mesure ou $\mathrm{X}$ est un signal et où ce signal peut être interprété comme le signal de quelque chose d'autre $»$ (p. 37). De correction en correction, l'Auteur aboutit à la conception suivante :

l'échange commence bien avant le premier signal et exige aussi plus qu'un simple B. Il n'y a pas que A, appelé l'émetteur, X, appelé le signal, et B, appelé le récepteur. Notre conducteur d'automobile, avant d'agir sur un commutateur, a froid ou chaud ou ne voit pas bien, etc., [...]: là est la source du circuit de l'échange. La mise en marche de A transforme celui-ci en transmetteur. Le signal a besoin d'un canal pour être transmis, qui sera ici un fil électrique mais qui dans d'autres cas pourra être une onde radio ou de la lumière. B demeure le récepteur mais est à distinguer du destinataire qui interprète le message que lui fournit le récepteur. Enfin, rappelons que le destinataire doit aussitôt, par le même cheminement ou par un autre, signaler à la source (pour laquelle cela constituera le feedback) que le signal s'est bien rendu et que le message a été bien interprété (p. 38).

Selon l'Auteur, cette description constitue un modèle adéquat de la communication, permettant d'analyser toute communication au sens large mais non dilué du terme; inversement, tout processus inanalysable par ce modèle ne sera pas de la véritable communication.

La troisième section porte sur la conversion du message en signaux. "Le message (ou l'information transmise) constitue la finalité de la communication»(p. 41); mais il n'est jamais transmis lui-même directement, il doit être transformé en 
éléments concrets qui lui serviront de support et de véhicule : les signaux. "Convertir un message en signaux véhiculaires et reconvertir, au bout de la transmission, ces signaux en le message initial, les deux opérations reposant sur un accord entre les deux êtres impliqués, c'est mettre en marche un code $\gg$ (p. 42). Le code est une organisation abstraite comprenant un lexique, c'est-à-dire "l'inventaire de toutes les unités utilisables dans les processus de signalisation ", un corps de règles syntaxiques "qui fixent les possibilités et impossibilités de combinaison parmi les unités physiques ", ainsi qu'un corps de règles sémantiques «qui déterminent les multiples rapports possibles entre les combinaisons d'unités physiques (de signaux) et les messages " (p. 42-43). L'utilisation d'un code comprend deux opérations symétriques: l'encodage et le décodage.

La quatrième section traite des bruits et des brouillages. Les accidents qui perturbent le processus de communication s'appellent des bruits, et ceux-ci ont des effets variés, pouvant aller jusqu'à l'opacité totale. Dans le cas de bruits produits volontairement dans un code et destinés à être reconnus comme tels et décodés par certains récepteurs privilégiés, on peut parler de brouillage; l'exemple le plus évident est celui des "messages codés" du monde de l'espionnage. Sont encore considérés comme bruits des phénomènes tels que «les voyages de masse où la collision des codes culturels se produit entre deux aéroports " (p. 51); la prise de conscience du fait que tel usage est culturel, et non naturel ; le remplacement progressif, en peinture, d'un original par sa reproduction, etc.

Comment diminuer les risques de bruit? Il faut d'abord, explique la dernière section, mesurer leur danger, mesure qui constitue l'objet de la théorie de l'information. Après avoir rappelé les rudiments de celle-ci, l'Auteur s'attarde à trois types de complications du codage qui peuvent contrecarrer les perturbations dues aux bruits: la multiplication des codes, c'est-à-dire "le fait qu'un émetteur, pour assurer que son message soit effectivement véhiculé jusqu'au récepteur et pour augmenter les chances qu'a le message d'être bien compris, convertit le message en signaux à l'aide de plusieurs codes à la fois " (p. 59), par exemple en ajoutant à un signal d'alarme 
lumineux un signal sonore; la cohérence ("Un message cohérent, c'est un message qui ne fait pas référence à des choses radicalement hétéroclites; c'est un message dont les parties se ressemblent, c'est-à-dire dont les parties sont, dans une certaine mesure, répétitives": p. 62); et enfin la répétition: " un message, ou une partie de message, est simplement communiqué deux fois" (p. 63). Ces trois moyens de contrer les bruits sont des phénomènes de redondance.

2.2. Cette seconde partie suscite beaucoup moins de remarques que la précédente. Nous nous contenterons de quelques commentaires portant sur le code. Il nous apparait tout d'abord étonnant que le code ne soit pas considéré comme un élément constitutif du modèle de la communication.

Par ailleurs, la notion de code nous semble quelque peu embrouillée. Le code, en effet, comprend d'une part un lexique, c'est-à-dire un inventaire des unités physiques (signaux) utilisables, d'autre part des règles syntaxiques fixant les possibilités de combinaison des signaux, et des règles sémantiques déterminant les rapports possibles entre les combinaisons de signaux et les messages. Or, selon l'Auteur, chaque message exige qu'on choisisse dans le lexique certaines unités plutôt que d'autres, ce qui implique qu'à chaque unité on ne peut attribuer qu'un nombre limité de valeurs extrinsèques :

En d'autres termes on ne peut pas affirmer que dans tous les systèmes de communication un message est véhiculé par une combinaison complète de signaux même en admettant que parfois signal et combinaison de signaux se confondent, car l'attribution préférentielle des signaux indique que ceux-ci possèdent déjà des bribes de messages potentiels ( $p$. 43)

Les mots, ajoute l'Auteur, peuvent être considérés comme les unités physiques inventoriées, c'est-à-dire comme lexique, dans ce code qu'est la langue. Mais les mots ont-ils un sens? Si l'on concède, par exemple, que voler veut dire "planer" ou "dérober" selon le contexte, on s'expose à se faire rétorquer que « ce n'est que dans la phrase (combinaison complète de signaux) que les mots (signaux) ont un sens (valeur extrinsèque) » (p. 43). À quoi l'on pourrait opposer des contre-exemples où le mot voler conserverait son ambiguité malgré le contexte, "ce qui prouverait que les valeurs 
extrinsèques sont stockés [sic !] dans le lexique, aux côtés des unités physiques » (p. 43). Contre-exemples qui en engendreraient d'autres, et ainsi de suite. L'Auteur fait alors intervenir la notion de double articulation :

c'est le fait fondamental pour le langage humain que non seulement on peut énoncer un nombre infini de messages par l'intermédiaire d'un nombre fini de mots-signaux [. . .], mais que ces mots-signaux, en nombre fini mais très élevé, sont décomposables à leur tour en un nombre très limité de signaux plus petits (les "phonèmes"). À ce niveau, évidemment, l'inventaire, le "lexique», se compose d'unités physiques dépourvus [sic !] de toute valeur extrinsèque et utilisables dans la construction de n'importe quels mots. Les seules contraintes combinatoires proviendront du corps de règles syntaxiques (p. 44).

Mais les phonèmes sont-ils des unités physiques? D'une manière générale, si l'on définit les signaux comme des unités physiques tout en admettant que les signaux comme tels relèvent de la physique et de la technologie plutôt que la sémiologie (p. 40), et que "la sémiotique doit s'articuler autour du signe, non du signal » (p. 51), ne s'ensuit-il pas que le lexique, et donc le code (en partie) ne relèvent pas de la sémiologie? Comment situer cette notion de signe par rapport au modèle de la communication, où n'interviennent que le signal et le message? Qu'en est-il de ces deux notions par rapport aux diverses conceptions du signe que nous avons rappelées plus haut et dont certaines (celle de Saussure par exemple) seront utilisées par l'Auteur dans la troisième partie? Si les mots possèdent des "bribes de messages potentiels", ne faudrait-il pas modifier la définition du lexique comme répertoire de signaux? La phrase est-elle vraiment une simple combinaison de signaux et ne faudrait-il pas tenir compte à son égard, comme à celui des mots, de la distinction entre sinsigne et signe (signifiant et signifié)? Des exemples d'ambiguïté équivalent-ils à des exemples d'absence de sens? Enfin, dans le cas des fameux "codes culturels", peut-on vraiment parler encore d'un lexique ainsi que de règles syntaxiques et sémantiques? À répondre à quelques-unes au moins de ces questions, l'ouvrage gagnerait en clarté, en précision et en cohérence. Le simple fait d'avouer, à propos de la définition du 
code, que " cette systématisation est vulnérable " (p. 43), ne nous apparaît pas suffisant.

\section{Les fondements de la sémiotique générale}

3.1. Cette troisième partie du volume résume la contribution des plus importants théoriciens de la sémiologie. Une première section est consacrée à Peirce, le philosophe américain qui est considéré, avec Saussure, comme le fondateur de la discipline. La seconde section porte sur la sémiologie saussurienne : l'Auteur rappelle les idées maîtresses de Saussure, puis la façon dont elles ont été reprises et élaborées par Hjelmslev et par Barthes. Enfin, sous l'angle des fonctions du langage, la dernière section évalue l'apport de Bühler, de Bloomfield et, plus longuement, de Jakobson.

Quelques pages sont consacrées en appendice à montrer comment le concept peircien d'icône peut être utilisé avec profit dans l'étude du langage.

3.2. "L'originalité, si j'ose parler ainsi, de mon introduction est de réduire au minimum l'archi-connu (Saussure, etc.) et d'accorder une place de choix au méconnu (Peirce, etc.) » (p. 12) : nous voudrions, pour terminer cette étude, prolonger ces préoccupations de l'Auteur en insistant sur un aspect particulier de la sémiologie peircienne : sa complexité.

3.2.1. «Peirce est source, il n'est que de puiser », écrivait, en conclusion d'un article consacré au philosophe américain, Gérard Deledalle ${ }^{37}$. Mais encore faudrait-il, en effectuant cette opération, ne pas utiliser une épuisette ! Si l'on considère la pensée sémiologique de Peirce, disséminée à travers les huit volumes de ses Collected Papers ${ }^{38}$, on doit en effet constater qu'elle a été souvent mal comprise par ses interprètes francophones. Voyons quelques exemples.

37. G. Deledalle, « Charles S. Peirce et les maîtres à penser de la philosophie européenne d'aujourd'hui ", in Études philosopbiques, 2(1964), p. 294.

38. Charles S. PEIRCE, Collected Papers, Cambridge: The Belknap Press of Harvard University, 1960, 1965 et 1966. Les six premiers volumes ont été publiés sous la direction de Charles Hartshorne et de Paul Weiss, et les deux derniers, sous celle d'Arthur W. Burks. Ces volumes ne reproduisent qu'une partie des textes écrits par Peirce. Pour une description de l'ensemble de ces textes, cf. Richard S. ROBIN, Annotated Catalogue of the Papers of Charles S. Peirce, The University of Massachusetts Press, 1967. 
En mettant en parallèle la démarche sémiotique et la démarche axiomatique, Julia Kristeva écrit : «En effet c'est à un des premiers axiomaticiens Charles Sanders Peirce qu'on doit l'emploi moderne du terme sémiotique ${ }^{39}$. 》Kristeva cite alors, en note, le texte suivant de Peirce :

La logique, dans son sens général est, je crois l'avoir montré, seulement un autre mot pour sémiotique ( $\sigma \eta \mu \epsilon \iota \omega \tau \iota \mu \hat{\eta})$, une doctrine quasi nécessaire ou formelle, des signes. En décrivant la doctrine comme "quasi nécessaire » ou formelle, j'ai en vue que nous observons les caractères de tels signes comme nous le pouvons, et à partir de telles observations, par un processus que je ne refuse pas d'appeler Abstraction, nous sommes amenés à des jugements éminemment faillibles, et par conséquent dans un sens absolument nécessaires, relatifs à ce que doivent être les caractères des signes utilisés par l'intelligence « scientifique»... (Philosophical Writings of Peirce, ed. by J. Buchler, 1955, p. 98).

Comment des jugements éminemment faillibles peuvent-ils être «par conséquent » absolument nécessaires? Une telle inférence, de la part d'un axiomaticien, serait pour le moins surprenante. Mais en fait Peirce parle de jugements faillibles et donc, en un sens, aucunement nécessaires ( and therefore in one sense by no means necessary »: 2.227); par ailleurs, le caractère « quasi nécessaire, ou formel », de la doctrine tient au processus d'abstraction qui lui permet de produire ses énoncés, et non à un quelconque souci de formalisation.

Second exemple. Dans un ouvrage de vulgarisation, Carontini et Peraya écrivent :

Rappelons tout d'abord que pour Peirce la science de la sémiotique se répartit en trois branches distinctes : la pragmatique qui implique le sujet parlant, la sémantique qui étudie le rapport entre le signe (representamen) et la chose signifiée (denotatum) ; enfin la syntaxe qui a pour objet la description des relations formelles entre les signes. ${ }^{40}$.

Or cette division de la sémiologie en trois branches a été proposée non par Peirce, mais par Charles Morris :

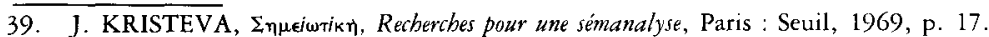

40. E. CARONTINI et D. PERAYA, Le projet sémiotique, Paris Jean-Pierre Delarge, 1975, p. 23-24. Carontini et Peraya citent aussi (p. 22) le même texte que Kristeva, et dans la même traduction erronée. On retrouve la même erreur dans l'ouvrage de Julia Joyaux : Le langage cet inconnu (Paris, S.G.P.P., 1969, p. 283). 
The relations of sign vehicles to what is designated or denoted may be called the semantical dimension of semiosis, and the study of this dimension semantics; the relation of sign vehicles to interpreters may be called the pragmatical dimension of semiosis, and the study of this dimension pragmatics; the remaining semiotically relevant relations of sign vehicles to other signs may be called the syntactical dimension of semiosis, and its study syntactics. Semiotic, as the general science of signs, thus contains the subordinate sciences of syntactics, semantics, and pragmatics $^{4}$.

Peirce distinguait bien, lui aussi, trois secteurs de la sémiologie, mais les nommait et les décrivait tout autrement :

It has three branches: 1 , Speculative Grammar, or the general theory of the nature and meanings of signs, whether they be icons, indices, or symbols ; 2, Critic, which classifies arguments and determines the validity and degree of force of each kind ; 3, Methodeutic, which studies the methods that ought to be pursued in the investigation, in the exposition, and in the application of truth (1.191).

Troisième exemple. Dans son Essai d'une philosophie $d u$ style, Gilles Gaston Granger, après avoir signalé que le schéma peut-être le plus suggestif du fonctionnement des signes (linguistiques) est celui de Peirce, cite, du philosophe américain, la définition suivante:

Un signe ou « representamen » est « une chose reliée sous un certain aspect à un second signe, son " objet », de telle manière qu'il mette en relation une troisième chose, son "interprétant", avec ce même objet, et ceci de façon à mettre en relation une quatrième chose avec cet objet, et ainsi de suite ad infinitum ... . ${ }^{42}$

\section{Voici le texte anglais de Peirce :}

A Sign is anything which is related to a second thing, its Object, in respect to a Quality, in such a way as to bring a

41. C. MORRIS, "Esthetics and the Theory of Signs ", in Writings on the General Theary of Signs, The Hague : Mouton, 1971, p. 417. Voir aussi : Foundations of the Theory of Signs, Chicago : The University of Chicago Press, 1938, p. 6-7. "Denotatum" est aussi un terme morrissien. Quelques-unes des citations de Peirce faites par Carontini et Peraya sont empruntées à l'article "Sémiotique " de O. DUCROT et T. TODOROV (Dictionnaire encyclopédique des sciences du langage, Paris : Stuil, 1972, p. 113 à 122); ceux-ci mentionnent les trois dimensions de la sémiologie, mais en les attribuant correctement à Morris.

42. G.-G. Granger, Essai d'une philosophie du style, Paris: Armand Colin, 1968, p. 114. 
Third thing, its Interpretant, into relation to the same Object, and that in such a way as to bring a Fourth into relation to that Object in the same form, ad infinitum (2.92).

Là où Peirce parle de l'objet comme d'une seconde chose (" a Second Thing "), Granger voit un second signe. Or si la thèse que l'objet est lui aussi un signe ne peut être confirmée par le texte que cite Granger, elle peut l'être, par contre, par d'autres textes du philosophe américain. Pour se faire une idée plus exacte de la conception peircienne du signe et prendre conscience des problèmes qu'elle pose, il faut donc tenir compte sinon de l'ensemble, du moins de la majorité de ses définitions du signe. C'est ce que nous nous proposons de faire maintenant.

3.2.2. Les définitions peirciennes du signe s'échelonnent sur plus de quarante années de production intellectuelle. Sans prétendre à l'exhaustivité, nous en examinerons une vingtaine. Nous les présenterons en fonction de leurs affinités, en trois groupes répartis entre autant de tableaux. Dans chaque cas, nous sous-entendons les mots « un signe, ou representamen ${ }^{43}$, est ... . Voici le premier groupe.

43. La plupart du temps, "signe ", et " representamen " sont, pour Peirce, équivalents. II lui arrive de faire une distinction entre les deux termes (v.g. 2.242), mais elle n'est pas nécessaire à notre propos. 


\begin{tabular}{|c|c|c|c|c|}
\hline A & relation conjointe & à la chose dénotée (à l'objet) & et à l'esprit & $\begin{array}{l}\text { 2. } 360-361 \\
(1885)\end{array}$ \\
\hline B & relation triadique & à son objet & et à son interprétant & $\begin{array}{l}\text { 1. } 292 \\
(1908)\end{array}$ \\
\hline $\mathrm{C}$ & $\begin{array}{l}\text { Triade authentique servant d'in- } \\
\text { termédiaire }\end{array}$ & entre un objet & et une pensée interprétante & $\begin{array}{l}1.480 \\
(1896)\end{array}$ \\
\hline $\mathrm{D}$ & $\begin{array}{l}\text { Chose ayant un caractère en vertu } \\
\text { duquel }\end{array}$ & $\begin{array}{l}\text { elle tient lieu d'une autre chose } \\
\text { (son objer) }\end{array}$ & $\begin{array}{l}\text { pour la production d'un certain } \\
\text { effet mental (son interprétant) }\end{array}$ & $\begin{array}{l}1.564 \\
(1899)\end{array}$ \\
\hline $\mathbf{E}$ & chose (a) & qui dénote un fait ou un objet (b) & pour une pensée interprétante (c) & $\begin{array}{l}1.346 \\
(1903)\end{array}$ \\
\hline $\mathrm{F}$ & $\begin{array}{l}\text { chose } \\
\text { en relation triadique }\end{array}$ & $\begin{array}{l}\text { déterminée par un objet } \\
\text { à son objet }\end{array}$ & $\begin{array}{l}\text { et qui détermine dans l'esprit } \\
\text { d'une personne une idée (inter- } \\
\text { prétant) médiatement déterminée } \\
\text { par l'objet } \\
\text { et à son interprétant }\end{array}$ & $\begin{array}{l}8.343 \\
(1908)\end{array}$ \\
\hline G & $\begin{array}{l}\text { Tente de représenter au moins en } \\
\text { partie }\end{array}$ & $\begin{array}{l}\text { un objet qui est cause, détermi- } \\
\text { nant, du signe, }\end{array}$ & $\begin{array}{l}\text { et effecte un esprit, y détermine } \\
\text { sous quelque rapport une chose } \\
\text { (l'Interprétant) qui est médiate- } \\
\text { ment due à l'objet }\end{array}$ & $\begin{array}{l}6.347 \\
(1909)\end{array}$ \\
\hline $\mathrm{H}$ & un connaissable & $\begin{array}{l}\text { déterminé par une autre chose } \\
\text { que soi (son objet) }\end{array}$ & $\begin{array}{l}\text { et qui détermine de telle façon un } \\
\text { Esprit actuel ou potentiel (cette } \\
\text { détermination étant l'interpré- } \\
\text { tant) que l'Esprit cst médiate- } \\
\text { ment déterminé par l'objet }\end{array}$ & $\begin{array}{l}8.177 \\
\text { s.d. }\end{array}$ \\
\hline
\end{tabular}


La parenté de ces huit définitions est évidente. Leur noyau commun stipule que le signe est une chose en relation triadique avec deux autres choses : un objet et un interprétant. À ce noyau se greffent les déterminations de l'objet et de l'interprétant. L'objet, ou chose dénotée, est autre chose que le signe ; celui-ci en tient lieu, le dénote, alors que lui-même le détermine, en est la cause. Quant à l'interprétant, c'est l'effet mental du signe sur l'esprit (actuel ou potentiel) d'une personne, c'est une pensée, une idée; il est déterminé immédiatement par le signe et médiatement par l'objet. En éliminant quelques redondances, et en laissant en réserve la caractérisation de l'interprétant, on peut conclure que, selon ce premier groupe de définitions, est signe toute chose qui est en relation triadique avec deux autres cboses, un objet et un interprétant, l'abjet dénoté déterminant le signe, qui détermine lui-même l'interprétant.

Passons maintenant au second groupe de définitions. 


\begin{tabular}{|c|c|c|c|c|c|}
\hline I & toute chose & qui réfere à un objet & $\begin{array}{l}\text { et en détermine une } \\
\text { autre (son interpréta- } \\
\operatorname{tant} \text { ) }\end{array}$ & $\begin{array}{l}\text { à référer de la même façon au } \\
\text { même objet, l'interprétant deve- } \\
\text { nant à son tour signe et ainsi de } \\
\text { suite à l'infini }\end{array}$ & $\begin{array}{l}\text { 2. } 303 \\
(1901)\end{array}$ \\
\hline $\mathrm{J}$ & $\begin{array}{l}\text { un premier en telle } \\
\text { relation triadique } \\
\text { authentique }\end{array}$ & $\begin{array}{l}\text { à un second (son } \\
\text { objet) }\end{array}$ & $\begin{array}{l}\text { qu'il est capable de } \\
\text { déterminer un Troi- } \\
\text { sième (son Interpré- } \\
\operatorname{tant)}\end{array}$ & $\begin{array}{l}\text { à assumer la même relation tria- } \\
\text { dique à son objet }\end{array}$ & $\begin{array}{l}\text { 2. } 274 \\
(1902)\end{array}$ \\
\hline $\mathrm{K}$ & $\begin{array}{l}\text { sujet d'une relation } \\
\text { triadique }\end{array}$ & $\begin{array}{l}\text { à un second (son } \\
\text { objet) }\end{array}$ & $\begin{array}{l}\text { pour un troisième } \\
\text { (son interprétant) }\end{array}$ & $\begin{array}{l}\text { relation telle que le representa- } \\
\text { men détermine son interprétant à } \\
\text { se tenir dans la même relation } \\
\text { triadique au même objet pour } \\
\text { quelque interprétant }\end{array}$ & $\begin{array}{l}1.541 \\
(1903)\end{array}$ \\
\hline $\mathrm{L}$ & $\begin{array}{l}\text { Premier Corrélat } \\
\text { d'une relation tria- } \\
\text { dique }\end{array}$ & $\begin{array}{l}\text { le second Corrélat } \\
\text { s'appelant son objet }\end{array}$ & $\begin{array}{l}\text { et le Troisième Cor- } \\
\text { rélat possible s'appe- } \\
\text { lant son Interprétant }\end{array}$ & $\begin{array}{l}\text { relation par laquelle l'Interprétant } \\
\text { possible est déterminé à être le } \\
\text { premier Corrélat de la même rela- } \\
\text { tion triadique au même Objet } \\
\text { pour quelque Interprétant pos- } \\
\text { sible }\end{array}$ & $\begin{array}{l}2.242 \\
(1903)\end{array}$ \\
\hline M & $\begin{array}{l}\text { un objet en relation } \\
\text { triadique }\end{array}$ & avec son objet & $\begin{array}{l}\text { et avec une pensée } \\
\text { interprétante (entre } \\
\text { lesquels il sert d'in- } \\
\text { termédiaire), elle- } \\
\text { même un signe, }\end{array}$ & $\begin{array}{l}\text { de façon à établir entre l'interpré- } \\
\text { tant et son objet une relation } \\
\text { correspondant à sa propre relation } \\
\text { à l'objet }\end{array}$ & $\begin{array}{l}8.332 \\
(1904)\end{array}$ \\
\hline $\mathbf{N}$ & $\begin{array}{l}\text { toute chose (de na- } \\
\text { ture essentiellement } \\
\text { triadique) qui }\end{array}$ & $\begin{array}{l}\text { étant déterminée par } \\
\text { un objet }\end{array}$ & $\begin{array}{l}\text { détermine une inter- } \\
\text { prétation }\end{array}$ & $\begin{array}{l}\text { à la détermination, par son entre- } \\
\text { mise, du même objet }\end{array}$ & $\begin{array}{l}4.531 \\
(1906)\end{array}$ \\
\hline
\end{tabular}


Les définitions de ce second groupe sont elles aussi manifestement apparentées. N'en considérons d'abord que les trois premières colonnes de constituants. Dans la première, l'idée de relation triadique est commune à toutes les formules sauf à la première (I), qui la contient cependant implicitement puisqu'elle articule les trois termes qui, dans les autres formules, constituent la relation triadique. Les expressions caractérisant le premier terme de cette relation sont : «toute chose », « un objet », le « sujet » (de la relation), «un premier», le «Premier Corrélat». Si l'on convient de remplacer «sujet» $(\mathrm{K})$ par «Premier Corrélat» $(\mathrm{L})$, il ne reste à choisir qu'entre les mots « chose » et «objet» d'une part, et «Premier (Corrélat)» d'autre part. Or les mots «chose " et «objet» sont non seulement vagues, mais ambigus, puisqu'ils peuvent désigner chacun des trois termes de la relation. Il est donc avantageux de leur substituer la notion de "Premier" ${ }^{44}$. La première colonne, dès lors, nous fournit l'expression "Premier d'une relation triadique». La deuxième colonne se réduit manifestement à l'idée d'un Second (l'Objet), qui détermine le Premier qui s'y réfere. Quant à la troisième colonne, elle met en scène un Troisième (l'Interpré$\tan$ ), qui est déterminé par le signe et qui est une pensée ${ }^{45}$. La synthèse de ces trois colonnes nous permet donc de définir le signe comme le Premier d'une relation triadique à un Second (son objet) et à un Troisième (son Interprétant), l'Objet dénoté déterminant le signe, qui détermine lui-même l'interprétant. Cette définition est fondamentalement identique à celle que nous a fournie la synthèse du premier tableau. Il nous reste cependant à rendre compte de la quatrième colonne du second tableau. Celle-ci nous apprend que l'interprétant engendré par le signe, devenant lui-même signe, dénote le même objet pour un autre interprétant, et ainsi de suite à l'infini. C'est cette précision supplémentaire qui caractérise les définitions du deuxième

\footnotetext{
44. Nous sous-entendons le mot "corrélat ", comme Peirce lui-même le fait habirueilement.

45. L'expression "idée interprétante " apparaît aussi dans le contexte de la définition I. Que l'interprétant soit lui-même un signe (M) : nous reviendrons sur ce sujet en étudiant la quatrième colonne. Quant à l'idée que le signe est intermédiaire entre l'objet et l'interprétant (M), elle est implicitement contenue dans l'affirmation que le signe est déterminé par l'objet et détermine l'interprétant, lequel n’est donc déterminé que médiatement par l'objet.
} 
groupe, par rapport à celles du premier. On pourrait supposer qu'entre le premier et le deuxième groupe, il y a une évolution de la conception peircienne du signe. Mais un simple coup d'œil aux dates respectives des diverses formules montre que, chronologiquement, elles se chevauchent. Il vaut donc mieux considérer les premières comme incomplètes, mais non incompatibles avec les secondes. Nous pouvons donc conclure que les quatorze formules que nous avons examinées sont toutes des variantes de la même définition, à savoir qu'un SIGNE est le Premier d'une relation triadique à un Second (son Objet) et à un Troisième (son Interprétant), l'Objet dénoté déterminant le Signe, qui détermine lui-même l'Interprétant à devenir signe en dénotant le même Objet pour un autre Interprétant, et ainsi de suité à l'infini. Cette formulation a le désavantage d'impliquer une distorsion dans l'ordre des déterminations. Selon le premier tableau et selon les trois premières colonnes du second tableau, c'est l'objet qui détermine le signe, et le signe qui détermine l'interprétant. Mais lorsque l'on greffe à cette première définition l'idée d'une chaîne infinie des interprétants, le signe subséquent, qui est l'interprétant du signe antérieur, est déterminé non par l'objet, mais par le signe antérieur lui-même. Si le signe antérieur devenait l'objet du signe subséquent, il n'y aurait pas de problème; mais tel n'est pas le cas, puisque Peirce affirme expressément que la chaîne signifiante est relative au même objet.

Le troisième groupe de définitions ne nous permettra malheureusement pas de résoudre ce problème. 


\begin{tabular}{|c|c|c|c|c|c|}
\hline $\mathrm{O}$ & chose & $\begin{array}{l}\text { qui tient lieu d'une } \\
\text { autre }\end{array}$ & & & $\begin{array}{l}7.355 \\
(1873)\end{array}$ \\
\hline $\mathrm{P}$ & un signe est triadique & car il dénote un sujet & $\begin{array}{l}\text { et signifie une forme de fait qu'il } \\
\text { met en connexion avec le premier }\end{array}$ & & $\begin{array}{l}6.344 \\
(1909)\end{array}$ \\
\hline $\mathrm{Q}$ & $\begin{array}{l}\text { chose qui, sous quel- } \\
\text { que rapport ou capa- } \\
\text { cité, en référence à } \\
\text { une sorte d'idée (fon- } \\
\text { dement) }\end{array}$ & $\begin{array}{l}\text { tient leur d'une autre } \\
\text { chose (son objet) }\end{array}$ & $\begin{array}{l}\text { pour quelqu'un dans l'esprit de } \\
\text { qui elle crée un signe équivalent } \\
\text { ou plus développé (l'interprétant) }\end{array}$ & & $\begin{array}{l}2.228 \\
(1897)\end{array}$ \\
\hline $\mathrm{R}$ & $\begin{array}{l}\text { Tout representamen } \\
\text { est relié à trois } \\
\text { choses: le fonde- } \\
\text { ment, }\end{array}$ & l'objet & l'interprétant & & 2. 229 \\
\hline $\mathrm{S}$ & $\begin{array}{l}\text { toute chose relié par } \\
\text { rapport à une Qualité }\end{array}$ & $\begin{array}{l}\text { à une Seconde chose, } \\
\text { son objet, }\end{array}$ & $\begin{array}{l}\text { de façon à mettre une Troisième } \\
\text { chose, son Interprétant, en rela- } \\
\text { tion avec le même objẹt, }\end{array}$ & $\begin{array}{l}\text { et ce de façon à en } \\
\text { mettre une Qua- } \\
\text { trième en relation } \\
\text { avec cet objet de la } \\
\text { même manière, ad } \\
\text { infinitum }\end{array}$ & $\begin{array}{l}2.92 \\
(1902)\end{array}$ \\
\hline $\mathrm{T}$ & $a$ & un objet & $\begin{array}{l}\text { et un Interprétant qui est ce que } \\
\text { le Signe produit dans le Quasi- } \\
\text { esprit qu'est l'Interprète en déter- } \\
\text { minant ce dernier à une sensa- } \\
\text { tion, un effort ou un signe, déter- } \\
\text { mination qui est l'Interprétant }\end{array}$ & & $\begin{array}{l}4.536 \\
(1906)\end{array}$ \\
\hline $\mathrm{U}$ & $\begin{array}{l}\text { Tient lieu.... } \\
\text { véhicule }\end{array}$ & $\begin{array}{l}\text { de quelque chose } \\
\text { (l'objet) }\end{array}$ & $\begin{array}{l}\text { pour l'idée qu'il produit ou modi- } \\
\text { fie (l'Interprétant) } \\
\text { amenant à l'esprit quelque chose } \\
\text { du dehors (la signification) }\end{array}$ & & $\begin{array}{l}\text { 1. } 339 \\
\text { s.d. }\end{array}$ \\
\hline
\end{tabular}


Les définitions de ce troisième groupe peuvent être considérées comme déviantes par rapport à celle que nous a fournie la synthèse des deux premiers. Essayons pourtant de voir si elles sont irréductibles. La définition $\mathrm{O}$, qui ne met en relation que deux éléments, la chose qui tient lieu et celle dont elle tient lieu, s'écarte de la conception triadique du signe; mais on peut la considérer comme incomplète par rapport à la définition $\mathrm{U}$, qui précise que le signe tient lieu de l'Objet pour l'Interprétant. Conclusion que corrobore le contexte même de la définition $O$. Peirce déclare en effet :

(. . .) even a simple idea is of intellectual value for us not for what it is but as standing for some object to which it relates. Now a thing which stands for another thing is a representation or sign (7. 355).

Expliquant ensuite qu'un signe doit avoir en lui-même des qualités qui servent à le distinguer, et une connexion physique réelle avec la chose qu'il signifie, il ajoute :

Whatever has these two characters is fit to become a sign. It is at least a symptom, but it is not actually a sign unless it is used as such ; that is unless it is interpreted to thought and addresses itself to some mind. As thought is itself a sign we may express this by saying that the sign must be interpreted as another sign (7. 356).

L'élément manquant, la pensée (l'interprétant), nous est donc fourni par le contexte. Par suite, la définition $O$ correspond aux définitions $A, B, C$ et $E$ du premier groupe et cesse de faire problème. La définition $\mathrm{P}$ mentionne un sujet dénoté (au lieu de l'Objet) et une forme de fait signifiée (au lieu de l'Interprétant); simple divergence de vocabulaire, et d'autant moins importante que cette définition fait partie de même texte que la définition $G$, qui la précise expressément. Les définitions $Q$ et $R$ posent un même problème, celui du "fondement" (ground); c'est sous quelque rapport ou capacité, en référence à une sorte d'idée, que le signe tient lieu de l'objet (Q) ; mais, ce disant, on introduit dans la définition du signe un quatrième élément (signe-fondement-objetinterprétant) qui va à l'encontre de la nature essentiellement triadique (selon Peirce) du signe. On remarquera que les deux définitions tétradiques datent de 1897 et que, à une exception 
près (définition S), Peirce n'utilisera plus par la suite cette formulation. Il y a en effet un moyen de contourner le problème et de rétablir la définition triadique en admettant que ce que l'on appelle l'Objet du signe n'est pas nécessairement le tout de l'objet. Telle semble bien être la solution privilégiée par Peirce : « a sign endeavours to represent, in part at least ${ }^{46}$, an Object » (6. 347). La définition $S$ pose deux problèmes : celui de la Qualité par rapport à laquelle le signe est relié à son Objet, et celui de la Quatrième chose que le signe met en relation avec l'Objet. Le sens du mot «qualité » dans cette définition est loin d'être clair, puisqu'elle provient d'un contexte où Peirce vient de présenter les trois catégories qu'il appelle cénopythagoricienne (à cause de leur référence aux nombres : cf. 2. 87) : l'Originalité, l'Obsistance et la Transuasion, et que « in the Obsistential aspect, Originality presents itself as a Quality, which is something which is such as it is, and is so free from Obsistence as not even to be self-identical or individual » (2.91); comme ce n'est pas encore le moment de présenter les trois catégories de Peirce, je supposerai que la Qualité en question fait référence au fondement que nous avons rencontré dans les définitions $Q$ et $R$ et est passible de la même solution ${ }^{47}$. Quant à la Quatrième chose, elle ne peut correspondre ici qu'à l'Interprétant du second signe qu'est l'interprétant du premier signe ; le premier signe, en effet, est en relation triadique avec son objet et son interprétant; or, ce premier interprétant devient signe par rapport au même objet et à un second interprétant, "lequel » devient une «quatrième chose » si l'on tient compte simultanément des deux signes dont chacun, pris séparément, est Premier par rapport à son Second et à son Troisième ; malgré la référence à une « quatrième chose », le signe reste donc, en tant que tel, une entité triadique, et la définition $S$ ne contredit pas celle que nous avons retenue. Conforme à la définition triadique du signe, la définition $T$ présente cependant une conception de l'interprétant comme étant une sensation, un effort ou un signe, conception qu'on peut rencontrer ailleurs dans l'œuvre de Peirce (par exemple, en 8. 332), mais qui est beaucoup

46. C'est moi qui souligne. Ce fragment provient de la définition $G$.

47. La "Qualité " n'étant pas présentée comme un quatrième élément, on peut supposer qu'elle a subi la même réduction que le fondement dans la définition $G$. 
moins fréquente que celle de l'interprétant comme pensée, et qui suscite des problèmes particuliers ${ }^{48}$ sur lesquels nous devrons revenir. La définition $U$, enfin, est conforme à la définition triadique dans la mesure où elle maintient que le signe tient lieu de l'Objet pour l'Interprétant, mais pourrait s'en écarter si l'affirmation que le signe est aussi un véhicule amenant à l'esprit quelque chose du dehors (la signification) se rapportait à un élément autre que l'Objet. Or le contexte de cette double définition est constitué par le fragmeni non daté que voici :

A sign stands for something to the idea which it produces or modifies. Or, it is a vehicle conveying into the mind something from without. That for which it stands is called its object ; that which it conveys, its meaning; and the idea to which it gives rise, its interpretant. The object of representation can be nothing but a representation of which the first representation is the interpretant. But an endless series of representations, each representing the one behind it, may be conceived to have an absolute object at its limit. The meaning of a representation can be nothing but a representation. In fact, it is nothing but the representation itself conceived as stripped of irrelevant clothing. But this clothing can never be completely stripped of ; it is only changed for something more diaphanous. So there is an infinite regression here. Finally, the interpretant is nothing but another representation to which the torch of truth is handed along ; and as representation, it has its interpretant again. Lo, another infinite series (1.339).

Ce texte n'est pas tout à fait lumineux. L'idée de signe, illustrant ici la catégorie de la Tertiarité, devrait impliquer trois éléments. Dans la première formule, il y a bien trois éléments : le signe, ce dont il tient lieu, et l'idée qu'il produit ou modifie. Mais dans la seconde formule, qui est présentée par un " ou » comme étant une autre façon de dire la même chose, il ne reste que deux éléments : le véhicule et ce qu'il transporte dans l'esprit. Or, si le véhicule correspond au signe, ce qu'il transporte ne correspond ni à ce dont le signe tient lieu ni à l'idée qu'il produit, puisque ces deux derniers facteurs sonr ensuite appelés l'objet et l'interprétant, tandis que ce qu'il

48. Par exemple, si l'interprétant est une sensation ou un effort, peut-il (doit-il) devenir signe par rapport à un autre interprétant, n'y a-t-il pas, en pareil cas, de chaîne infinie des interprétants? 
transporte est nommé signification. Il faudrait donc tenir compte de quatre éléments : le signe, l'objet, l'interprétant et la signification. Mais peut-on encore, après cela, concevoir le signe comme une Tertiarité ? La suite du texte complique encore davantage les choses en affirmant que l'objet, et la signification, et l'interprétant constituent eux aussi des représentations. Si l'on revient aux deux formules initiales, il faudrait donc comprendre qu'un signe, une représentation, tient lieu d'une représentation pour la représentation qu'elle produit ou modifie en transportant dans l'esprit une représentation de l'extérieur. Comme si cela ne suffisait pas, il faut en outre admettre que les trois représentations que constituent l'objet, l'interprétant et la signification appartiennent chacune à une série infinie. Nous savions déjà que l'interprétant appartient à une série infinie, ce qui impliquait une série infinie de signes puisque chaque interprétant devient un signe par rapport à un autre interprétant; mais, et le signe et l'interprétant étaient en relation avec le même objet, et non avec une série infinie d'objets. La définition $U$ semble donc bien irréductible à la définition peircienne habituelle du signe. De plus si le signe est lui-même une représentation, et si toute représentation présuppose une représentation antérieure, on peut se demander comment un signe pourrait être une chose concrète (v.g. une girouette), et comment le processus sémiologique peut s'enclencher.

Des vingt et une définitions que nous avons passées en revue, deux seulement ( $\mathrm{T}$ et $\mathrm{U}$ ) s'écartent significativement et de façon apparemment irréductible des autres. Mais celles-ci posent également un certain nombre de problèmes. En fait, pour comprendre davantage la conception peircienne du signe, il nous faudrait savoir.

- ce que sont la Primarité, la Secondarité et la Tertiarité ;

- si nous avons eu raison de réduire la notion de fondement et celle de qualité ;

- si l'interprétant peut être non seulement une pensée, mais aussi une sensation ou un effort ;

- en quoi consiste la chaîne infinie des interprétants ; 

interprétant ;

quel est le rapport entre signification (définition $\mathrm{U}$ ) et

- comment il faut interpréter l'idée d'une chaîne infinie des objets, qui seraient eux aussi des représentations;

- quel sens donner à la distinction que fera assez tardivement Peirce entre deux objets et trois interprétants.

Demandons-nous, pour terminer, dans quelle mesure l'ouvrage de Jurgen Pesot peut nous aider à résoudre ces problèmes.

3.2.3. À la sémiologie peircienne, l'Auteur consacre une section (p. 71-93) qui traite respectivement de la triade et des catégories phénoménologiques, du signe et de la sémiose illimitée, ainsi que de la classification des signes. Seules nous intéressent ici les deux premières sous-sections.

Dans la première, l'Auteur commence par rappeler le caractère constamment triadique de toutes les conceptions peirciennes, caractère justifié par le fait que la triade est irréductible à l'unité ou à la paire, tandis que tout nombre supérieur à trois peut être obtenu à partir de trois ; c'est ainsi que le fait de donner, qui est une relation entre un donneur, une chose donnée et un receveur, est irréductible aux paires ou aux combinaisons de paires (donneur-chose donnée, chose donnée-receveur, donneur-receveur) en lesquelles on pourrait tenter de l'analyser, tandis que des raffinements de cette relation, comme la relation de vendre, se ramènent à une combinaison de relations triadiques. Après avoir précisé la notion du trois authentique, on peut aborder la question des catégories phénoménologiques (on phanéroscopiques). Une catégorie peut "se définir comme un mode d'être des choses, que celles-ci soient réelles ou non $»(p .75)$. Peirce nomme ses catégories la priméité, la secondéité et la tiercéitét ${ }^{4}$.

49. Cette traduction, qui est celle de Deledalle (dans C.S. PEIRCE, Érits sur le signe, Paris : Seuil, 1978), nous semble peu euphonique; nous lui substituerons, sauf dans les citations de Pesor ou de Deledalle, les termes Primarité, Secondarité et Tertiarité, qu'utilise Charles Bouton dans La signification. Contribution à une linguistique de la parole, Paris : Klincksieck, 1979, p. 45 ; (dans cet ouvrage de 332 pages, à peine plus d'une page est consacrée à Peirce, dont on affirme que "dans un certain sens, [il] annonçait Saussure " (p. 45) !) Sur le choix de "Priméité " (etc.) de préférence à "Primarité " (etc.), Deledalle s'est expliqué dans Théorie et pratique du signe. Introdution à la sémiotique de Charles S. Peirce, Paris : Payot, 1979, p. 57. 
Un « premier » sera ainsi le premier élément d'une priméité, d'une secondéité ou d'une tiercéité. Un "second", qui présuppose un premier, sera le second élément d'une priméité, d'une secondéité ou d'une tiercéité, Un « troisième», qui présuppose un premier et un second, sera le troisième élément d'une priméité, d'une secondéité ou d'une tiercéité (p. 75).

À quoi ces catégories correspondent-elles ? Voici deux réponses de Peirce à cette question :

Ce sont l'être de la possibilité qualitative positive, l'être du fait actuel, et l'être de la loi qui gouvernera les faits dans le futur.

La priméité est le mode d'être de ce qui est tel qu'il est, positivement et sans référence à quoi que ce soit d'autre. La secondéité est le mode d'être de ce qui est tel qu'il est par rapport à un second, mais sans considération d'un troisième quel qu'il soit. La tiercéité est le mode d'être de ce qui est tel qu'il est, en mettant en relation réciproque un second et un troisième $e^{50}$.

De chaque catégorie, l'Auteur donne quelques exemples dont voici un échantillon : Relèveraient de la Primarité le sentiment inanalysé, la couleur rouge d'un objet qui pourrait exister, etc. ; de la secondarité, des phénomènes comme l'expérience, l'existence réelle d'un objet, etc.; et de la tertiarité, les classes, les lois, les modèles dont les phénomènes seconds peuvent être des occurrences. Les premiers ne peuvent produire des seconds ni des troisièmes, mais ceux-ci impliquent des seconds qui impliquent des premiers. "Seuls les premiers peuvent être absolument premiers. Dès qu'il y a second, le premier est intégré dans une secondéité, c'est-à-dire que le second est second par rapport à une autre chose qui est son premier. De façon analogue, un troisième repose sur un second et un premier en ce qu'il instaure la relation entre ceux-ci " (p. 76).

Ces précisions étaient indispensables pour aborder le signe, car " le signe peircien est un troisième » (p. 77). Mais l'Auteur ajoute :

50. Textes cités p. 75-76. Dans les Collected Papers, le premier texte se trouve en 1.23 et le second en 8.328. Pour le premier, l'Auteur téfere à la traduction de Deledalle (p. 69) et pour le second, qui est pourtant lui aussi traduit par Deledalle, à la correspondance de Peirce, sans plus de précision 
En fait, dans la phraséologie de Peirce lui-même, la chose est un peu moins claire : "Un signe est une sorte de troisième " (Peirce 1978, p. 30). Ailleurs, seulement un des trois éléments du signe, l'interprétant, est considéré comme troisième. Le mot «signe " est aussi souvent utilisé à la place de "representamen» qui, pourtant, est un premier dont l'« objet » est le second (p. 77).

À son avis, cependant, si la terminologie, et surtout le mot signe, sont ambigus, la théorie, par contre, est cohérente et relativement univoque: "le signe est une relation triadique entre un premier, appelé le representamen, un second, appelé son objet, et un troisième, appelé son interprétant» $(\mathrm{p} .77)^{51}$. Le representamen, "c'est la facette du signe qui nous est directement accessible, c'est ce que l'utilisateur du signe sent, perçoit ou pense $\gg$ (p. 77); si le representamen est une primarité, il sera une qualité possible positive ; s'il est une secondarité, il sera un objet individuel, une réaction, un fait palpable et unique ; tandis que s'il est une tertiarité, il sera loi, concept, pensée. «L'objet n'est objet que s'il est l'objet (second) de quelque chose (premier). En tant que second, il peut avoir tous les modes d'être : si le second ressemble au premier, il s'agit d'une priméité ; si la relation entre le second et le premier est une relation de fait, comme un contact ou une relation de cause à effet, on a une secondéité ; enfin, si la relation n'est qu'instituée ou du moins appuyée par une sorte d'habitude ou de convention, on a affaire à une tiercéité " (p. 77-78). Quant à l'interprétant, s'il est primarité, c'est comme une interprétation possible, un terme, un verbe isolé de son contexte; $s^{\prime}$ il est secondarité, il sera proposition prononcée en un lieu et en un moment déterminés ; et s'il est tertiarité, il sera argument, raisonnement, déduction. L'interaction de ces paramètres

51. Cette "solution" n'est-elle pas trop optimiste? Considérons les propositions suivantes: La tiercéité est le mode d'être de ce qui est tel qu'il est, en mettant en relation réciproque un second et un troisième; (p. 76); un troisième repose sur un second et un premier en ce qu'il instaure la relation entre ceux-ci (p. 76); un signe est une sorte de troisième (p. 77) ; I'interprétant est un troisième (p. 77) « a sign mediates between the interpretant sign and its object " (8.332). Le problème n'est pas seulement de lever l'ambiguité entre le signe comme troisième (au sens de relation triadique) et l'interprétant connue troisième élément de cette relation, mais encore de savoir si la relation est instaurée entre un second et un troisième ou entre un premier et un second, et surtout d'expliquer comment, si la médiation est le fait du troisième et si l'interprétant est troisième, le signe peut être à la fois le premier de la relation triadique et ce qui instaure la médiation entre interprétant et objet. 
engendre une multitude de signes. "Le flou noté ci-devant n'en est plus un : "signe" peut être utilisé à la place de « representamen » si l'on envisage le signe en soi ; ce faisant, on a recours à une synecdoque tout à fait licite du même genre que celle qui permet d'évoquer tel «linguiste " qui est aussi «Français» et «professeur»» (p. 78). L'Auteur rappelle alors la définition peircienne du signe qui est le plus fréquemment citée $^{52}$, puis ajoute que le caractère triadique est à tel point essentiel dans la définition du signe que la notion de triade et celle de signe semblent parfois recouvrir le même champ: Peirce ne disait-il pas qu'il ne voyait que des signes en et autour de lui ? «En particulier, toute pensée est signe et a, par conséquent, un caractère triadique » (p. 79-80). En effet, la pensée est un monologue silencieux, le monologue est un dialogue entre deux instances de la même personne, et dialoguer, c'est émettre et interpréter des signes. L'on touche ainsi à la définition pragmatique de la sémiotique comme " doctrine de la nature essentielle et des variétés fondamentales de semiosis possibles » (p. 80). La semiosis n'est pas un processus de communication au sens où l'on a défini celui-ci plus haut, car elle met l'accent sur l'interprétation : «Elle instaure un face-à-face entre un signe et un interprète, non entre un émetteur et un récepteur ${ }^{53}$." Dans la semiosis, l'intention est une notion non pas psychologique, mais logique : elle fait intervenir une intelligence, une pensée, une tertiarité, donc un signe; ce signe, en tant qu'il est généré par un autre signe, est un interprétant :

La notion d'interprétant se présente finalement comme la cheville ouvrière de la sémiose: d'une part, l'interprétant garantit le caractère triadique du signe et le distingue ainsi des procès dyadiques; d'autre part, vu qu'il est par définition lui-même un signe, c'est lui qui inscrit toute pensée, toute parole, tout agir dans un processus sémiotique. Il a le même

52. Cf. supra, définition $Q$.

53. P. 80. On peut se demander pourquoi, en ce cas, l'Auteur n'a pas tenu compte de la position de Peirce en présentant les diverses tendances de la sémiologie. On notera d'ailleurs que l'affirmation que la semiosis n'est pas un processus de communication est doublement contestable. D'une part, en effet, si la pensée est un dialogue, l'interprète est à la fois émetteur et récepteur. D'autre part, si le concept de signe est plus général que celui de signal (signe servant à communiquer), une sémiologie des signes n'est pas une sémiologie de la communication non parce qu'elle exclut celle-ci, mais parce qu'elle l'englobe dans un champ plus vaste. 
objet que le signe dont il est l'interprétant. La chaîne sémiotique qui est ainsi créée par l'interprétant qui est à la fois résultat d'une première sémiose (où il est interprétant à proprement parler) et source d'une deuxième (où il agit comme representamen) équivaut à une sémiose illimitée (p. 80-81).

Pour résumer cette sémiosis illimitée, l'Auteur cite la définition I, puis l'illustre de la façon suivante: "L'interprétant est la traduction d'un signe en un autre signe du même code ou d'un code différent» (p. 81); ainsi, le signe forêt a pour objet l'ensemble d'arbres que nous nommons forêt; mais si nous savons cela, c'est qu'en chacun de nous se trouve une sorte de lexique où sont cataloguées les définitions des mots, dont celle du mot forêt ; or une telle définition est un signe qui s'appelle interprétant; à la place de cette définition pourraient se trouver un synonyme (bois), une image, une mimique, un mot équivalent dans une autre langue (wood, wald, etc.), et tous ces interprétants renvoient "au même objet que le signe dont il est la traduction" (p. 82). Mais un signe, en tant que representamen, peut renvoyer à plusieurs interprétants différents, et un interprétant, en tant que signe, peut renvoyer à plusieurs objets : "On voit maintenant aisément combien la chaîne de la sémiose illimitée est complexe " (p. 82).

3.2.4. Plusieurs commentateurs ${ }^{54}$ soulignent la difficulté de la théorie peircienne des signes (et parfois de sa conception des catégories), certains n'hésitant pas à la qualifier de fragmentaire, d'obscure, de confuse et même d'inconsistante. L'une des sources de cette difficulté est sans contredit le rapport étroit entre cette théorie des signes et la conception peircienne des catégories. Une autre vient du fait que la théorie peircienne des signes n'a pas poussé d'un seul jet. Selon Deledalle, en effet, on peut

distribuer les écrits de Peirce sur les signes en trois périodes: une période kantienne (1851-1870) où la théorie des signes est

54. Cf. Par exemple: A. Burks, P. Weiss, "Peirce's Sixty-six Signs", in The Journd of Philosophy, XLII (1945) 14, p. 383 ; A. Burks, "Icon, Index, Symbol ", in Phillossphy and Phenomenological Research, IX (1949) 4, p. 675; J. Fitzgetald, Peirce's Theory of Signs as Foundation for Pragmatism, The Hague: Mouton, 1966, p. 48 ; T. Goudge, The Thought of C.S. Peire, Toronto: Univ. of Toronto Press, 1950, p. 137; D. Greenlee, "Peirce's Hypostatic and Factorial Categories", in Transactions of Charles S. Peirce Society (TCSPS dans les références ultérieures), IV (1968) 1, p. 49 ; D. Greeniee, Peircés Concept of Sign, The Hague: Mouton, 1973, p. 8, 34, 134. 
liée à une révision des catégories kantiennes dans le contexte d'une logique aristotélicienne bivalente ou plus exactement dyadique; une période logique (1870-1887) au cours de laquelle Peirce propose, pour remplacer la logique aristotélicienne, une nouvelle logique, la logique des relations qui sera la base et le garant de sa conception triadique des catégories et des signes; une période sémiotique enfin (1887-1914) où Peirce développe sa nouvelle théorie des signes en liaison avec sa nouvelle théorie des catégories ${ }^{55}$.

Dans son introduction à la sémiologie peircienne, Deledalle s'en tient expressément aux textes de la troisième période. De même, les textes de Peirce qu'il a présentés dans Écrits sur le signe $^{56}$ relèvent eux aussi de cette période. Or l'Auteur, dans la mesure où les textes traduits par Deledalle constituent son unique source, s'impose la même limitation chronologique. Mais celle-ci est-elle justifiée? Si la logique des relations, élaborée au cours de la seconde période, constitue « la base et le garant de [la] conception triadique des catégories et des signes », et si « $\log i c$, in its general sense, is, as I believe I have shown, only another name for semiotic » (2.227), peut-on vraiment faire l'économie de la seconde période 57 ? Peut-on vraiment distinguer une période «logique » et une période « sémiotique »?

Venons-en pourtant à la description des catégories. L'Auteur présente ces dernières comme "un mode d'être des choses, que celles-ci soient réelles ou non ». Or, une telle définition est pour le moins inhabituelle. La plupart des commentateurs, en effet, rappellent que les catégories sont relatives au «phanéron», et décrivent celui-ci en des termes

55. G. Deledalle, Théorie et pratique da signe, p. 12-13.

56. C.S. Peirce, Écrits sur le signe (rassemblés, traduits et commentés par Gérard Deledalle), Paris : Seuil, 1978.

57. La même question pourrait être posée à propos de la première période, si l'on tient compte de ce qu'affirme Buzelli à propos de l'arricle "On a New List of Categories" : "The categories, as developed in 1867 , were never ractically changed thereafter. As to the method of their derivation, Peirce states that "my paper of 1867 was perhaps the least unsatisfactory, from a logical point of view, that I ever succeeded in producing ; and for a long time most of the modifications I attempted of it only led me further wrong (2.340, dated c. 1895). As late as 1893 he incended to include it in his proposed books on logic. Thus, this paper is a fundational one for Peirce's entire philosophy, and the argument by which the categories are developed is of no small value if one wishes to determine how he understood them "(D. Buzelli, "The Argument of Peirce's 'New List of Categories' ", in TCSPS, VIII (1977) 2, p. 63). 
analogues aux suivants : "Tout ce qui, à quelque point de vue et en quelque sens que ce soit, est présent à l'esprit de qui que ce soit, partout et toujours, qu'il corresponde ou non à quelque chose $^{58}$. " Le phanéron étant ce qui est présent à l'esprit, les catégories correspondent aux différents aspects que l'on peut y observer. Là où l'Auteur parlait de mode d'être des choses, il faudrait donc parler de modalité des idées. La situation est encore plus complexe que cela; comme le souligne en effet Ayer en parlant des catégories, Peirce "refers to them sometimes as Modes of Being and sometimes as Ideas" ". Murphey, de son côté, souligne qu'il y a, des catégories, un aspect formel ainsi qu'un aspect matériel, ce qui suscite des difficultés :

But it is one of the most ubiquitous sources of confusion in Peirce's writing that in respect to the status of concepts the material and formal aspects of the categories do not agree. Formally considered, concepts are monadic predicates; it would therefore appear that they must be Firsts. Yet materially considered concepts are not Firsts but Thirds. The result is at best lack of clarity and at worst downright inconsistency $(\text {. . . })^{60}$

Quant à Greenlee, il parle d'une conception hypostatique et d'une conception factorielle des catégories :

As modes of beings or classifications of things, the categories are hypostatic. As classes of factors discovered in the analysis of a subject matter to which the categories are applied, a category is factorial ${ }^{61}$.

Thus in an expression such as the "Firstness of Secondness ", "Secondness " is to be taken to represent a kind of phenomenon, a mode of being, whereas "Firstness" is to be taken to represent an aspect or factor of that phenomenon ${ }^{62}$.

Si, en effet, les catégories n'étaient que des modes d'être des choses, quel sens y aurait-il à affirmer, comme le fait

58. G. Deledalle, Théorie et pratique du signe, p. 22.

59. A.J. Ayer, The Origins of Pragmatism, San Francisco: Freeman and Cooper, 1968, p. 101. On notera toutefois que la caractérisation des catégories en termes de modes d'être est présentée par Peirce dans le contexte du classement des idées (8.328).

60. M. Murphey, The Development of Peirce's Philosophy, Cambridge: Harvard University Press, 1961, p. 308-309.

61. Peirce's Concept of Sign, p. 40.

62. "Peirce's Hypostatic and Factorial categories", in TCSPS, p. 58. 
l'Auteur : «Un «premier» sera ainsi le premier élément d'une priméité, d'une secondéité ou d'une tiercéité ${ }^{63}$ " ?

Abordons maintenant la notion de signe. Que le signe soit triadique, Peirce l'affirme sans cesse, l'Auteur le souligne, et en cela il est d'accord avec la plupart des commentateurs. Mais pour se faire une idée de la complexité de cette conception triadique, on peut la comparer aux types de conceptions que nous avons présentés plus haut. Convenons, pour simplifier la discussion, que la conception pentadique inclut la conception tétradique, qui elle-même inclut la conception triadique. La conception pentadique articule, on s'en souvient, le sinsigne, le signifiant, le signifié, l'état de conscience et le désigné64 ${ }^{4}$; or, dans une version peircienne de cette conception, chacun de ses éléments pourrait être appelé un signe ! Si l'on préfere une comparaison dont les termes soient plus homologues, que l'on se rabatte sur la conception classique (signe-pensée-chose), et que l'on soit prêt également à admettre que chacun de ses éléments est lui-même un signe $^{65}$. Mais cette équivalence serait encore trop simple. Nous avons vu plus haut que l'Auteur, en décrivant le premier de la relation triadique (le representamen), affirmait qu'il peut lui-même être une primarité (une qualité. possible positive), une secondarité (un objet individuel, une réaction, un fait palpable et unique), ou une tertiarité (une loi, un concept, une pensée); en termes peirciens, il s'agit du qualisigne, du

63. P. 75. On notera par ailleurs que l'argument voulant qu'il n'y ait que trois catégories parce que toute relation plus complexe que la triade est réductible à des triades est incompatible avec la thèse à l'effet que les catégories résultent d'une observation du phanéron.

64. Certains de ces éléments, tels le sinsigne, relèvent, chez Peirce, non de la définition, mais de la classification des signes.

65. En un certain sens, on pourrait donc soutenir que le signe est une monade. C'est ce que laissent également entendre les définitions où le signe est défini comme le Premier de la relation triadique ( $c f$. définitions $\mathrm{J}-\mathrm{L}$ ) ou comme une chose qui est en rapport avec un objet et un interprétant (définitions D-E-F-I-M-N-O-Q-S). C'est par sa relation avec les deux autres éléments que le signe est triadique (l'Auteur, à ce sujer, parlait de " synecdoque »); de même, dans la conception saussurienne, le signe en lui-même est une dyade, et c'est par sa relation avec les autres éléments qu'il devient tétradique. C'est pourquoi il nous semble illégitime d'opposer, sans plus de précisions, comme le fait Deledalle (Théorie et pratique du signe, p. 34-35), la conception "dyadique " de Saussure à la conception «triadique " de Peirce. 
sinsigne et du légisigne; schématiquement, dans le cas du légisigne, on aurait :

$\sum_{1=2}^{3}={ }_{\text {(representamen) }}^{\text {I }}$ (objet) $\quad$ (interprétant)

Si l'on se rappelle par ailleurs que Peirce en était venu à poser une distinction entre deux objets et trois interprétants, cela signifie que, pour bien comprendre un légisigne, il faudrait tenir compte de huit éléments (de sept dans le cas du sinsigne, de six dans celui du qualisigne) : en quel sens, par rapport à de tels cas, peut-on affirmer que le signe est une relation triadique?

Mais qu'en est-il de ce double objet et de ce triple interprétant auxquels nous venons de faire allusion? L'Auteur ne s'en préoccupant guère, il nous faudrait, pour comprendre ces distinctions, recourir à d'autres sources. A titre illustratif, nous nous en tiendrons à l'objet du signe.

À ce propos, l'Auteur nous apprend, comme nous l'avons vu, que l'objet est second par rapport à un premier et que, en tant que second, il peut avoir tous les modes d'être, c'est-à-dire être une primarité, une secondarité ou une tertiarité. Mais cette dernière remarque concerne en fait la relation entre le signe et son objet et commande la fameuse division des signes en icône, indice et symbole : il reste à préciser ce qu'est l'objet, et cette tâche n'est rien moins qu'aisée. Les différentes expressions utilisées dans les définitions que nous avons présentées plus haut - (ce qui détermine le signe, ce que le signe dénote, ce à quoi il se réfere, ce dont il tient lieu, la chose dénotée) - laissent le problème intact. Dans son lexique des principaux termes peirciens, Deledalle écrit :

OBJET [Object]. Tout ce - quel qu'il soit, réel ou imaginaire - à quoi l'interprétant renvoie le representamen. Le terme "référant " pourrait lui convenir ${ }^{66}$.

Mais si l'on admet que le référent est habituellement une chose actuellement existante, l'objet sera un " référent " d'autant plus spécial qu'il peut être non seulement une chose imaginaire

66. Théorie et pratique du stgne, p. 22. 
mais encore une chose qui, tout en étant réelle (v.g. une loi, généralité ${ }^{67}$ relevant de la tertiarité), n'existe pourtant pas (ce qui existe relève, selon Peirce, de la secondarité). On ne s'étonnera donc pas que les interprétations de l'objet varient grandement d'un commentateur à l'autre. Ces interprétations peuvent être classées en trois groupes, selon qu'elles se fondent sur la synchronie, sur la panchronie ou sur la diachronie ${ }^{68}$.

Les deux ouvrages que Deledalle a consacrés à la sémiologie peircienne de la période 1887-1914 illustrent très bien l'approche synchronique de l'objet ${ }^{69}$. Le premier volume présente surtout, à propos de l'objet, une mosaïque de citations développant les points suivants :

- Le signe est mis pour un objet indépendant de lui, mais cet objet doit être de la nature d'un signe (d'une pensée) puisqu'il est censé affecter le signe (1.538).

- L'objet est nécessaire, sinon aucune information ne serait communicable (2.231), mais que cet objet corresponde ou non à quelque chose de réel est hors de question ; en tout cas, cela n'est pas du ressort de la grammaire spéculative $(1.284)^{70}$.

- Le mot signe sera employé pour dénoter un objet perceptible, imaginable ou même inimaginable $(2.230)^{71}$.

- Le signe représente un objet mais ne le fait ni connaittre ni reconnaître : l'objet doit déjà être connu (2.231).

- Il y a deux façons pour un symbole d'avoir pour objet réel

67. Sur les rapports entre la réalité des généralités selon Peirce et le réalisme de Duns Scot, cf. C.K. McKeon, "Peirce's Scotistic Realism ", in Studies in the Pbilosopby of Charles Sanders Peirce (éd. par P. Wiener et F. Young), Cambridge : Harvard University Press, 1952, p. 238-250.

68. Au besoin, on pourrait subdiviser ces trois groupes selon que les commentateurs utilisent ou non les manuscrits inédits.

69. Cf. Érits sur le signe, p. 216 et 224-229; Théorie et pratique du signe, p. 22, 65-70 et p. $118-120$.

70. Le paragraphe 1.284 n'a pas trait à la grammaire spéculative, qui est une partie de la sémiologie, mais à la phanéroscopie, science antérieure à la sémiologie.

71. En dépit de l'emploi du verbe dénoter et du mot objet, l'objet dont il est question dans cette phrase n'est pas l'objet du signe mais le signe comme objet, ainsi que le montrent à la fois les exemples utilisés et le mouvement du texte, qui caractérise d'abord le signe en lui-même, puis l'objet: "The word sign will be used to denote an Object perceptible, or only imaginable, or even unimaginable in one sense - for the word "fast ", which is a sign, is not imaginable, since it is not this uord itself that can be set down on paper or pronounced, but only an instance of it, and since it is the very same word when it is written as when it is pronounced, but is one word when it means " rapidly" and quite another when it means "immovable", and a third when it refers to abstinence. But in order that anything should be a sign, it must " represent ", as we say, something else, called its Object $» \ldots$. 
une chose existentielle réelle : la chose peut s'y conformer, accidentellement ou parce que le symbole a la vertu d'une habitude croissante, ou encore le symbole comprend un indice comme partie de lui-même; mais l'objet immédiat d'un symbole ne peut être qu'un autre symbole, et s'il a par nature un autre genre d'objet, ce doit être par une série infinie (2.293 n. 1).

- L'objet d'un signe varie selon que le signe est premier, second ou troisième. Le premier est "l'objet comme le signe lui-même le représente», le second est un «objet efficient, mais non immédiatement présent " (8.343); le troisième est un symbole. Le premier est immédiat, le deuxième dynamique $(8.343)^{72}$.

- L'objet d'un signe n'est ni l'interprétant ni sa propre signification ${ }^{73}$, mais la chose ou l'occasion, aussi indéfinie soit-elle, à laquelle on va l'appliquer, sa signification étant l'idée qu'il attache à cet objet par supposition, décision ou affirmation (5.6).

Dans le second ouvrage, Deledalle reprend l'idée que l'objet doit être un signe et qu'il doit déjà être connu, puisque le signe ne le fait ni connaître ni reconnaître. Il ajoute que l'objet, puisqu'il est second, est " ce à quoi le signe renvoie dans sa singularité existentielle ${ }^{74}$ » et qui n'est pas nécessairement une chose, un événement ou une signification puisque, comme l'écrivait Peirce à Lady Welby, le mot objet désigne tout ce qui vient à la pensée ou à l'esprit. Le signe est un représentant de l'objet. Mais l'objet, comme nous le savons déjà, est double, et Deledalle précise cette distinction comme suit :

L'objet immédiat est l'objet tel que le signe le représente; l'objet dynamique est l'objet réel «que, par la nature des

72. Voici le texte de Peirce: " A Sign, therefore, has a triadic relation to its Object and to its Interpretant. But it is necessary to distinguish the Immediate Object, or the Object as the sign represents it, from the Dynamical Object, or really efficient but not immediately present Object. " Il y a deux objets, non trois. Comme le précisait le texte précédent (2.293 n. 1), le symbole qui joue le rôle d'un objet est un objet immédiat.

73. La position de Peirce, à propos de la signification, r'est pas aussi limpide : "A Symbol is a law, or regularity of the indefinite future. Its Interpretant must be of the same description ; and so must be also the complete immediate Object, or meaning " $(2.293$; c'est moi qui souligne; c'est sur cette phrase que se greffe la note citée plus haut disant que l'objet immédiat d'un symbole est un symbole).

74. Deledalle utilise ici la secondarité de façon hypostatique plutôt que factorielle : or, comment concilier la présente affirmation et ce qui a été dit plus haut de l'objet du symbole? 
choses, le signe ne peut pas exprimer, qu'il ne peut qu'indiquer en laissant à l'interprète le soin de la découvrir par expérience collatérale $»(8.314)^{75}$.

Deux textes développent cette distinction. Selon le premier, l'objet immédiat n'est pas tout l'objet : il en est un élément ; le signe ne peut renvoyer [...] d'une manière significative que si l'objet total est déjà connu» $(8.177 \mathrm{n}, 4)^{76}$; par exemple, celui qui dit que Napoléon était léthargique a l'esprit déterminé par Napoléon, sinon il ne pourrait y penser, et l'interprète doit être déterminé par l'objet de cette phrase grâce à une expérience collatérale indépendante de l'action du signe, sinon il ne sera pas déterminé à penser à cet objet; or, Napoléon ne peut déterminer son esprit que si le mot attire son attention sur la bonne personne, ce qui n'est possible que si l'interprète possède déjà une habitude telle que le mot suggère un certain nombre d'attributs de l'homme Napoléon ; et, dans la phrase citée, «Napoléon» n'est qu'un objet partiel, "léthargie " en est un autre ; un objet peut dès lors déterminer un signe trompeur ou erroné, et le signe peut donner l'existence à l'objet : l'objet de "Napoléon " est l'univers de l'existence dans la mesure où il est déterminé par le fait que Napoléon en est membre, tandis que l'objet de la phrase "Hamlet était fou " est l'univers de la création shakespearienne dans la mesure où il est déterminé par le fait qu'Hamlet en fait partie (8.178). Quant au second texte, il pose que l'objet immédiat est l'objet en tant que tel, " dans le signe ", et que l'objet dynamique est l'objet « hors du signe ", dans son contexte, ce dernier n'apparaissant pas immédiatement dans les representamens : le signe doit suggérer son objet dynamique ou "médiat », et cette suggestion ou sa substance est l'objet immédiat; ainsi dans la proposition

75. Cité p. 22. L'expression " objet réel " n'est pas très heureuse puisque, dans le passage auquel Deledalle se réfere, Peirce décide de parler de l'objet dynamique plutôt que de l'objet réel parce que l'objet pourrait être fictif

76. La note en question précise le sens du mot objet dans la définition $H$. Dans cette définition, il n'est pas question de l'objet immédiat au sens où l'on vient de le définir, mais du fait que l'objet détermine médiatement l'esprit interprétant. Voici maintenant la note : " or, in some cases, as if the sign be the sentence 'Cain killed Abel', in which Cain and Abel are equally Partial Objects, it may be more convenient to say that that which determines the Sign is the Complexus, or Totality, of partial Objects. And in every case the Object is accurately the Universe of which the special Object is member of part. " Peirce ne dit pas que l'objet immédiat est une partie de l'objet, mais que, dans le cas de signes (complexes) comme une phrase, l'objet est lui aussi complexe. 
" le soleil est bleu », les objets sont « le soleil» et « la bleuité "; si par "bleuité " on entend l'objet immédiat, la qualité de la sensation, il ne peut être connu que par le sentiment, mais si l'on entend cette condition existentielle réelle qui fait que la lumière émise a une longueur d'onde moyenne courte, Langley a prouvé que la proposition est vraie; de même le "soleil » peut signifier l'occasion de sensations brûlantes (objet immédiat) ou l'interprétation ordinaire de ces sensations en termes de lieu, de masse, etc., (objet dynamique) ; mais si quelqu'un montre le soleil du doigt en disant : "Voilà ce que nous appelons le soleil ", le "soleil " n'est pas l'objet de ce signe, c'est le signe du soleil, le mot "soleil ", qui en est l'objet, et l'on ne peut savoir de quoi il est le signe que par une expérience collatérale dont le soleil sera l'objet immédiat et dynamique $(8.183)^{77}$. En résumé, selon Deladalle, l'objet est quelque chose qui est de la nature d'un signe et qui doit déjà être connu par l'interprète grâce à une expérience collatérale ; en tant qu'objet immédiat, il est l'objet tel que le signe le représente, un élément de l'objet total, l'objet en tant que tel, l'objet dans le signe ; en tant qu'objet dynamique, il est ce que le signe ne peut qu'indiquer et qu'il faut découvrir par expérience collatérale, l'objet hors du signe, dans son contexte ${ }^{78}$.

77. L'exemple "Le soleil est bleu " est précédé, dans le texte de Peirce, de ceci, qui n'est pas sans importance pour la compréhension de la notion d'objet : "As to the Object, that may mean the Object as cognized in the Sign and therefore an idea, or it may be the Object as it is regardless of any parricular aspect of it, the Object in such relations as unlimited and final study would show it to be. The former I call the Immediate Object, the latter the Dynamical Object. For the latter is the Object that Dynamical science (or what at this day would be called "Objective " science,) can investigate " (8.183). $\dot{A}$ propos de l'exemple cité, Ayer, pour qui la difficulté que l'on éprouve à décrire de façon cohérente la théorie peircienne des signes vient de l'obscurité de sa notion d'objer (The Origins of Pragmatism, p. 156), fait le commentaire suivant : "One difficulty is that according to Peirce's definition, the immediate object is not a different object from the dynamical, but the same object differently conceived ; but while there is no difficulty in holding that the sun which is the occasion of sensation, is the same object as the sun to which we ascribe the property of mass, the two bluenesses are so far from being identical that the choice between them makes a difference to the truth-value of the proposition. To say that the sun is blue is false if the reference is to the quality of a sensation, but true, according to Peirce, if the reference is to the condition which causes the emitted light to have a short wave-lenght. But what that implies is that the word "blue " is ambiguous, in the sense that under different interpretations it refers to different objects, rather than that it refers to the same object in different aspects " (p. 132-133).

78. Selon Ayer (opus cit., p. 156), l'objet dynamique correspond à ce quauparavant Pierce appelait le fondement du signe ; cette interprétation peut être confirmée par le fait que 
Les interprétations panchroniques de la théorie peircienne du signe en général, et de l'objet du signe en particulier, abondent. Nous mettrons ici l'accent sur celle de Greenlee ${ }^{79}$. La thèse majeure de Greenlee est que l'idée qu'un signe représente un objet, en tient lieu, en est le substitut, est trop étroite pour rendre compte du fonctionnement de tous les signes : par exemple, le signe qui se représente lui-même (tel point sur la carte d'une île représentant le même point sur l'île elle-même), ou encore un signe comme « et», qui ne tient lieu d'aucun objet, seraient laissés pour compte. Cette critique n'empêche cependant pas Greenlee d'expliquer en quoi consiste, pour Peirce, l'objet du signe. À propos de la distinction entre objet immédiat et objet dynamique, il reprend certaines définitions que nous avons déjà rencontrées chez Deledalle: l'objet immédiat est l'objet tel qu'il est représenté dans le signe, tandis que l'objet dynamique est celui que le signe ne peut exprimer, qu'il peut seulement indiquer en laissant à l'interprète le soin de le trouver par une expérience collatérale; celui-ci est la chose représentée telle qu'elle est en elle-même, indépendamment de sa relation à la pensée, tandis que celui-là est la même chose, mais en relation avec la pensée. Cette distinction peut être reportée au niveau de la pensée si l'on considère que la pensée d'un moment, en tant qu'elle influence dynamiquement celle d'un moment ultérieur, peut être assimilée à un objet dynamique (7.353); en ce cas, l'objet immédiat serait le moment antérieur en tant que représenté dans le moment ultérieur. Greenlee rappelle aussi que le signe ne peut pas faire connaître l'objet. Par exemple, les empreintes d'un tigre ne seront signe, pour un chasseur, que si celui-ci connaît déjà la connexion entre le tigre et ses empreintes; en ce cas, l'objet dynamique est le tigre qui a laissé les traces, tandis que l'objet immédiat est le tigre en tant que représenté par ces traces interprétées par le chasseur. Greenlee rappelle encore que, pour Peirce, dans une triade

la division des signes en icône, indice et symbole, d'abord esquissée par rapport au fondement, le sera plus tard par rapport à l'objet dynamique. Dès lors, n'est-il pas erroné de prétendre, comme le fait Yvan Almeida (L'opératinité sémantique des récits-paraboles, Louvain : Éd. Peeters, et Paris : Éd. du Cerf, 1978, p. 61-62 et 74), que le fondement est un facteur non structural situé " en dehors du système des catégories sémiotiques"?

79. Peirce's Concept of Sign, p. 51-69 et 107-114. 
authentique, l'objet est lui aussi un signe, mais il ajoute que cette conception entraîne, des objets, une régression à l'infini telle que la connaissance ne pourrait jamais commencer. $\grave{A}$ cette objection Peirce aurait répondu par sa théorie de la connaissance (1868-1869), en montrant que toute connaissance est processus et interprétation et que toute connaissance particulière peut être indéfiniment analysée en n'importe quel nombre de connaissances antérieures et postérieures, toutes mutuellement reliées selon la relation du signe qui détermine l'interprétant ${ }^{80}$. Mais, en dépit de toutes ces explications, Greenlee n'en maintient pas moins que l'objet n'est pas toujours nécessaire à l'existence d'un signe. Cette thèse a évidemment suscité de multiples réactions. C'est ainsi qu'Oehler, dans un compte rendu ${ }^{81}$, déclare, en s'appuyant sur un texte inédit de Peirce, que l'objet immédiat se trouve dans l'intention du locuteur, qu'il est une idée, une appréhension, un ens rationis ; l'objet immédiat peut exister sans l'objet réel, mais non inversement ; l'objet est nécessaire au signe, mais il sera objet immédiat s'il s'agit de l'idée sur laquelle le signe est construit, et objet réel s'il s'agit de la chose réelle, de la circonstance sur laquelle cette idée se fonde ; l'objet immédiat

80. L'argument de Peirce (5.262-263) repose sur l'idée que la série de connaissances est continue et s'apparente au paradoxe d'Achille. Voici comment Ayer (opus cit., p. 105) commente ce passage : " Peirce tries to show that the case of his series of cognitions is analogous by introducing the simile of an inverted triangle which is partly dipped in water. The apex of the triangle represents the object of the cognitions. The cognitions themselves are represented by the lines made by the surface of the water across the triangle, the earlier cognitions corresponding to the lower lines. Then, since any line must be separated by a finite distance from the apex, there is no lowest line.

But while this is an effective illustration, it is a bad analogy. It is true that a process which lasts for any length of time has infinitely many phases, in the sense that any stretch of it can be infinitely subdivided, but this is not to say that it consists of an infinite number of processes of the same kind as itself. The mere fact, which Peirce strangely adduces as an argument in his favour, that cognitions are not instantaneous tells conclusively against him : for if there is a lower limit to the time during which a process of cognition can take place, it follows that, even if it can be represented as having an infinite number of parts, these parts cannot themselves all be cognitions. The fact that the time which it takes is infinitely subdivisible no more implies that the cognition can be so divided than the fact that the distance which Achilles covers in his first step is infinitely subdivisible implies that he thereby takes an infinite number of steps. "

Pour une interprétation du même passage, plus sympathique à Peirce, cf. $\mathrm{J}$ Buchler, Charles Sanders Peirce's Empiricism, New York: Octagon Books, 1966, p. 16-18; pour une discussion plus détaillée de la théorie peircienne de la connaissance en 1868, cf. D. Savan, "On the Origins of Peirce's Phenonenology ", in Studies in the Philosophy of Charles Sanders Peirce (éd. par Wiener et Young), p. 185-194.

81. Compte rendu publié dans TCSPS, X (1974) 3, p. 185-189. 
est subjectif, c'est une idée qui résulte de l'observation collatérale aidée par l'imagination et la pensée. Fitzgerald ${ }^{82}$, de son côté, soutient que Peirce utilise les mots signe, objet et interprétant de manière analogique; ainsi, dans le cas de l'objet, l'objet immédiat est l'idée sur laquelle le signe est construit, tandis que l'objet réel est la chose ou la circonstance sur laquelle le signe est construit mais qui n'apparaît pas dans le signe; par exemple, lorsqu'une girouette indique la direction du vent, celle-ci n'apparaît pas dans le signe : l'objet réel du signe est le vent, mais la connaissance de celui-ci est présupposée par l'utilisation de la girouette; le mot objet a donc une double acception; l'objet réel (ou dynamique) est celui qui est le plus en vue quand on utilise le signe dans des contextes courants :

That is what the sign stands against in the primary sense of that term. However, closer analysis of a sign reveals to us that there is something internal to the sign which relates it to the thing or circumstances. To bring out this ground and its relation to the dynamic object, as well as to the mode of discovery, the term " object " is extended to apply to it ${ }^{83}$.

Ransdell ${ }^{84}$, enfin, pour montrer que Greenlee a eu tort de supprimer, de la définition du signe, l'objet, rappelle que Peirce a modelé la triade objet-signe-interprétant sur la triade énonciateur-énoncé-interprétation, l'objet dérivant de l'énonciateur parce que c'est la réalité qui est l'énonciateur ultime des signes, qui parle à travers les hommes; Ransdell rappelle également que, puisque Peirce nie l'existence de l'intuition, l'interprétation de tout phénomène (de tout signe) présuppose une interprétation antérieure prise pour acquise à ce moment : or, l'ensemble de ces interprétations antérieures est l'objet tel qu'on croit le connaître, l'objet immédiat, qui differe de l'objet réel, qui est l'objet tel qu'il est réellement, indépendamment des idées qu'on en a à tel moment, indépendamment de sa représentation dans l'objet immédiat ; cela ne signifie pas que l'objet réel soit une sorte de chose en soi inconnaissable : il

82. J. Fitzgerald, "Ambiguity in Peirce's Theory of Signs", in TCSPS, XII (1976) 2, p. $127-134$.

83. Art. cit., p. 132

84. J. Ransdell, "Another interpretation of Peirce's Semiotic ", in TCSPS, XII (1976) 2, p. $97-110$. 
est ce qui apparaît dans l'objet immédiat quand il est interprété correctement, il est identique à ce qu'il serait connu être dans une interprétation idéale ultime où l'objet immédiat coïnciderait avec l'objet réel ; celui-ci n'est donc pas nécessaire à toute semiosis, alors que celui-là l'est. En s'appuyant sur d'autres documents tout aussi inédits, Greenlee ${ }^{85}$ rétorque que l'objet réel ne saurait être l'opinion ultime, l'interprétation correcte et totale du signe, parce que celle-ci est un idéal, une possibilité future, alors que l'objet réel est un objet dynamique, quelque chose qui existe (au sens peircien) actuellement : l'objet réel est quelque chose que l'interprète doit déjà connaître, quelque chose qui agit dyadiquement sur le signe et lui est antécédent: l'objet, cause déterminante du signe, lui est antérieur, alors que l'interprétant (la signification), étant déterminé par le signe, lui est subséquent :

The worst mistake to make, which, admittedly, some of Peirce's confusing formulations about the object do encourage, is to equate the ultimate opinion with the real or dynamical object. The correct understanding of the matter is that the real object is that which that opinion represents and which, even in the case of currently used signs that carry the truth-value of truth, is represented by those signs. The real object is not that final ideal opinion but is rather what, in Peirce's words to Lady Welby, " the object in such relations as unlimited and final study would show it to be " $(8.183)^{86}$.

L'adoption d'un point de vue diachronique permet-elle de départager cette multitude d'interprétations opposées? Murphey, dans l'ouvrage qu'il a consacré au développement de la philosophie peircienne, fait d'abord état d'un premier système (1859-1861) reconstitué à partir d'inédits de jeunesse, très fortement influencé par la problématique kantienne. C'est ainsi que la théorie peircienne de l'objet de la représentation dériverait de la théorie kantienne de l'objet transcendantal:

All representations have, as representations, their object, and can themselves in turn become objects of other representations. Appearances are the sole objects which can be given to us immediately, and that in them which relates immediately to

85. D. Greenlee, "Peirce's Concept of Sign : Further Reflections", in TCSPS, XII (1976) 2, p. $135-147$.

86. Ast. cit., p. 146 
the object is called intuition. But these appearances are not things in themselves; they are only representations, which in turn have their object - an object which cannot itself be intuited by us ...87

Murphey mentionne à ce propos qu'il y a alors, dans la pensée de Peirce, une ambiguïté fondamentale, qui allait le hanter encore pendant des années :

Peirce's early thought is antiphenomenalistic in that he rejects Kant's division between the phenomenal object and the Ding an sich and holds that we do know things as they really are. To this extent Peirce is a realist. But he also holds that whatever is, is thought-of, so that nothing exists apart from its relation to the mind, and [. . . ] that things must be as our knowledge represents them to be, because the constitution of our minds forbids their ever being known to be otherwise. These arguments are phenomenalistic — indeed, idealistic ${ }^{88}$.

En retraçant les origines du second système peircien, Murphey s'attarde ensuite longuement sur un célèbre article de 1867 : «On a New List of Categories » (1.545-559) ainsi que sur les ébauches qui l'ont précédé. Dans cet article, Peirce développe l'idée que, pour que la substance soit unie à l'être dans un jugement perceptuel chargé de ramener à l'unité la diversité des impressions sensibles, trois conceptions (ou catégories) sont nécessaires : celles de la qualité, de la relation et de la représentation. La qualité, c'est ce qui incorpore une abstraction; celle-ci est nécessaire parce qu'on ne peut comprendre l'accord entre deux choses que comme un accord selon un certain point de vue, lequel constitue une abstraction (v.g. la noircéité) ; celle-ci peut être appelée un fondement, et la référence à un fondement constitue une qualité, un attribut général ; la qualité existe donc à un niveau d'abstraction inférieur à celui de la propriété abstraite. Mais on ne peut connaître une qualité que par le fait qu'elle est semblable à une autre ou différente d'elle ; or, par la similitude et la différence, une chose se réfere à un corrélat : c'est donc à l'occasion de la référence à un corrélat que l'on introduit la référence à un fondement, et c'est la comparaison qui est l'occasion de la

\footnotetext{
87. A $108 f$; cité par Murphey, opus cit., p. 29 ; la référence aux inédits est faite par rapport à la boîte pertinente à la bibliothèque.

88. P. $40-41$.
} 
référence à un corrélat ; mais cette comparaison implique que l'on tienne compte de deux objets, car on ne peut savoir qu'un objet possède une qualité qu'en le comparant à une autre qualité ; 'de plus, la comparaison ne peut s'instituer qu'en référence à un interprétant au niveau de la représentation :

every comparison requires, besides the related thing, the ground and the correlate, also a mediating representation which represents the relate to be a representation of the same correlate which this mediating representation itself represents. Such a mediating representation, I call an interpretant, because it fulfills the office of an interpreter who says that a foreigner says the same thing which he himself says ${ }^{89}$.

C'est dans la théorie de la connaissance contemporaine de cette élaboration des catégories que Peirce soutient que, toute connaissance étant déterminée par une connaissance antérieure, l'objet est toujours une représentation, qu'il n'y a pas de connaissance initiale et que l'on ne peut posséder de connaissance directe d'un objet externe. Mais si, à partir de 1870 , Peirce s'est efforcé de modifier sa théorie de la connaissance, ce n'est qu'à partir de 1885 qu'il a entrepris une révision de ses catégories :

Peirce's theory of reality had run into serious difficulties in the late 1870 's. This theory of reality is a direct result of the categorial theory of the "New List " and the cognitive theory of the 1868 papers. By denying the existence of the first impressions of sense, Peirce had completely sundered the real from perception, so that direct acquaintance with reality cannot be gained by going to the source of our cognitions. The only alternative left therefore was to locate the real at the end of the series of cognitions by defining reality as that which is thought in the final opinion to which inquiry will lead. But Peirce then found himself unable to prove that in fact inquiry will ever lead to any final result and accordingly unable to prove that there is any reality. As a result Peirce's position degenerates into an extreme form of subjectivism in which we are lost in a phantasmagoric maze of our concepts. For one who called himself a realist, such a development was intolerable ${ }^{90}$.

Comment transformer cette situation? En renonçant à l'idée que la réalité est ce qui sera pensé dans l'opinion ultime, ainsi

89. D4́p 12f. Cité : p. 83.

90. Opus cit., p. 301. 
qu'à celle qui lie la réalité à un chercheur (collectif) éternel, "we may now regard the real as depending upon the coherence of observations ${ }^{91} »$, ce qui implique une révision substantielle et de la théorie peircienne des catégories, et de sa théorie de la connaissance: car si le réel est ce qui donne cohérence à l'expérience, il est nécessaire, pour échapper au conceptualisme pur, que ce à quoi la cohérence est conférée comporte autre chose que des éléments purement conceptuels. Désormais, les catégories ne seront plus dérivées de l'analyse du signe, comme cela avait été le cas en 1867, mais équivaudront à trois sortes de relations logiques : monadiques, dyadiques et triadiques; de plus, le contenu de ces relations devra être tiré de l'expérience. En particulier, alors que, dans l'article de 1867 , l'objet lui-même ne pouvait jamais être immédiatement connu, dans la nouvelle conception, la désignation directe d'un objet existant individuel ${ }^{92}$ deviendra possible grâce au concept d'baecceity, qui correspond à l'expérience du choc, de la résistance, de la réaction brutale, etc ${ }^{93}$.

3.2.5. Qu'est-ce que l'objet d'un signe? À cette question, non seulement les commentateurs ne répondent pas de la même façon, mais, comme nous venons aussi de le constater, Peirce lui-même apporte des réponses différentes à différentes époques de sa carrière. On trouverait autant de divergences à propos de la notion d'interprétant. Par exemple, combien y a-t-il d'interprétants? Dans la plupart des définitions du signe que nous avons recensées plus haut, il est question d'un seul interprétant (membre d'une chaîne infinie). Mais, en d'autres textes, il est question de trois interprétants: l'interprétant immédiat, l'interprétant dynamique et l'interprétant final (v.g. $4.536 ; 8.184 ; 8.314 ; 8.315)$; l'interprétant final est aussi appelé l'interprétant « normal » $(8.343)$; par ailleurs, il

91. lbid., p. 303 .

92. Cela n'entraine pas que l'objet soit extérieur : cf. Murphey, opus cit., p. 310-311.

93. Murphey étudie encore une "ultime révision (1896-1914) " des catégories, où l'accent est mis sur l'introduction d'une nouvelle science, la phénoménologie, pour tendre compte des catégories ; nous ne nous y arrêterons pas parce que " as actually practiced, Peirce's phenomenology differs in no respect from the doctrine of the caregories as he had presented it since 1885 \%(p. 367). À l'égard de l'introduction de cette nouvelle discipline, Murphey est d'ailleurs très sévère : "It is impossible to regard Peirce's phenomenological treatment of the categories as anything more than a quite unsuccesful sleight of hand $》$ (p. 368). 
est également question d'un interprétant émotionnel, d'un interprétant énergétique et d'un interprétant logique (5.475476). Selon certains auteurs, ces deux triades sont identiques :

Peirce distingue trois sortes d'interprétants: immédiat [immediate], dynamique [dynamical] et final [final] ou normal [normal]. L'interprétant immédiat est l'interprétant représenté dans le signe, l'interprétant dynamique est l'effet réel que le signe produit sur l'esprit, l'interprétant final ou normal est une habitude - l'habitude acquise par expérience de renvoyer un certain type de representamen à un certain type d'objet. Ces trois interprétants, vus du côté de l'interprète, sont dits affectif [emotional], énergétique [energetic] et logique [logical ${ }^{94}$.

Fitzgerald, par contre, soutient que la seconde triade d'interprétants n'est pas identique à la première mais qu'elle doit être considérée comme une subdivision de l'interprétant dynamique ${ }^{95}$. On pourrait encore s'interroger sur la chaîne infinie des interprétants et se demander si elle est relative à un même objet, comme le laissaient entendre les définitions que nous avons citées, ou à une pluralité d'objets, comme le pense Yvan Almeida ${ }^{96}$. Et quel est le rapport entre la notion d'interprétant et celle de signification ${ }^{97}$. Et ce qu'il en est de l'interprétation pragmatique de l'interprétant. Etc.

Sans doute, dans un bref ouvrage d'initiation à la sémiologie, ne pouvait-on s'attendre à ce que l'Auteur résolût tous ces problèmes. Mais on aurait pu espérer qu'il indiquât clairement que "l'essentiel " auquel il réduisait la sémiologie peircienne était prélevé sur une période précise (1887-1914) de la pensée du philosophe plutôt que sur l'ensemble de sa philosophie. De plus, sans résoudre tous ces problèmes dont nous avons seulement donné un échantillon, l'Auteur aurait à tout le moins pu indiquer qu'il y a, dans la sémiologie

94. G. Deledalle, Théorie et pratique du signe, p. 22.

95. J. Fitzgerald, Peirce's Theory of Signs as Foundation for Pragmatism, p. 16-83.

96. Y. Almeida, L'opérativité sémantique des récits-paraboles, p. 54-60.

97. À propos de la définition $U$, nous avons noté plus haut qu'elle semblait tétradique dans la mesure où elle distinguait le signe, l'objet, l'interprétant et la signification. Selon Justus Buchler (opus cit., p. 109-111), le mot objet désignerait, dans ce passage, l'objet immédiat, tandis que les mots interprétant et signification désigneraient deux facettes de l'interprétant logique; car "signification" (au sens étroit) et "interprétant logique " seraient, pour Peirce, synonymes ; Murphey (opus cit., p. 314-316) n'est pas d'accord : la signification, dit-il, n'est pas l'interprétant, mais une habitude. 
peircienne, des problèmes, ainsi que les enjeux de ceux-ci. À trop simplifier la théorie peircienne des signes, on risque d'en conférer au lecteur une fausse maîtrise qui l'empêchera de pousser plus loin ses investigations, ce qui n'était sûrement pas l'intention de l'Auteur, car celui-ci, reconnaissant que "beaucoup de thèmes ont été abordés à la manière d'un petit voleur dérobant ici une pomme, là une gomme (p. 143) ", conclut en formulant "l'espoir que ce livre aura su donner à l'un le goût, à l'autre le savoir nécessaires pour aller chercher dans d'autres livres ou par lui-même ce que le présent livre n'aura pas eu l'heur de lui fournir (p. 144) ».

Faculté de philosophie

Université Laval 